\title{
Uniqueness and asymptotic behavior of solutions with boundary blow-up for a class of nonlinear elliptic equations
}

by

\author{
M. MARCUS \\ Department of Mathematics, \\ Israel Institute of Technology-Technion, 32000 Haïfa, Israël. \\ and

\section{VÉRON} \\ Département de Mathématiques, Faculté des Sciences et Techniques, \\ Parc de Grandmont, 37200 Tours, France.
}

\begin{abstract}
We study the uniqueness and expansion properties of the positive solutions $u$ of $(E) \Delta u+h u-k u^{p}=0$ in a non-smooth domain $\Omega$, subject to the condition $u(x) \rightarrow \infty$ when dist $(x, \partial \Omega) \rightarrow 0$, where $h$ and $k$ are continuous functions in $\bar{\Omega}, k>0$ and $p>1$. When $\partial \Omega$ has the local graph property, we prove that the solution is unique. When $\partial \Omega$ has a singularity of conical or wedge-like type, we give the asymptotic behavior of $u$. When $\partial \Omega$ has a re-entrant cuspidal singularity, we prove that the rate of blow-up may not be of the same order as in the previous more regular cases.
\end{abstract}

Key word: Nonlinear elliptic equations.

RÉSUMÉ. - Nous étudions les propriétés d'unicité et de comportement limite des solutions positives $u$ de $(E) \Delta u+h u-k u^{p}=0$ dans un domaine non régulier $\Omega$, sujettes à la condition $u(x) \rightarrow \infty$ quand dist $(x, \partial \Omega) \rightarrow 0$, où $h$ et $k$ sont des fonctions continues dans $\bar{\Omega}, k>0$ et $p>1$. Quand $\partial \Omega$

A.M.S. classification: $35 \mathrm{~J} 60$.

Annales de l'hstitut Henri Poincaré - Analyse non linéaire - 0294-1449 
a la propriété du graphe local, nous démontrons que la solution est unique. Quand $\partial \Omega$ a une singularité de type conique ou dièdrale, nous donnons le comportement asymptotique de $u$. Quand $\partial \Omega$ a une singularité cuspide rentrante, nous montrons que l'ordre de l'explosion peut ne pas être le même que dans les cas précédents.

\section{O. INTRODUCTION}

In this paper we study positive solutions of the problem

$$
\begin{aligned}
\Delta u+h u-k u^{p} & =-f \quad \text { in } \Omega . \\
u(x) \rightarrow \infty \quad \text { as } \delta(x) & =\operatorname{dist}(x, \partial \Omega) \rightarrow 0
\end{aligned}
$$

where $\Omega$ is a domain in $\mathbb{R}^{N}$ or $S^{N}$ (= the unit sphere in $\mathbb{R}^{N+1}$ ) with (possibly) non-smooth boundary. Here $N \geq 2, p>1$ and $h, f, k$ are continuous functions in $\bar{\Omega}$ with $k>0$ and $f \geq 0$. In order to simplify the presentation, we shall confine our discussion to bounded domains. However the results can be extended to unbounded domains with compact boundary and also to some classes of domains with unbounded boundary. In fact, positive solutions of the problem in unbounded conical domains play an important role in the present study.

The mains topics treated in this paper are, existence and uniqueness, rate of blow-up of solutions at the boundary and a principle of localization which is central to our investigation. Our main interest is in the study of these problems in domains with non-smooth boundary. An uniqueness result in domains with non-smooth boundary was recently established by Le Gall [LG] (by probabilistic methods) in the case $p=2, h=0$ and $k=1$. In the case of domains with smooth boundary i.e. boundary of class $C^{2}$, existence uniqueness and rate of blow-up of solutions for problem $(0.1),(0.2)$ (and also for more general equations) have been thoroughly investigated (see [BM1,2,4] and [V1] and the references cited there). The existence and uniqueness results of the present paper can also be extended to a larger class of problems, similar to the one treated in [BM4]. These and other extensions will be discussed elsewhere.

We turn now to a brief description of the main results.

(i) Existence. If $\Omega$ is a domain satisfying the exterior cone condition then Problem (0.1), (0.2) possesses a maximal and a minimal positive solution. (Corollary 1.10). 
(ii) Uniqueness. If $\Omega$ satisfies the local graph property then Problem $(0.1)$, (0.2) has at most one solution. (See Theorem 2.2).

A domain $\Omega$ satisfies the local graph property if every point $P \in \partial \Omega$ has a neighborhood $Q_{P}$ and a local set of Cartesian coordinates $\xi$ with origin at ${ }^{\prime}$, such that $\Omega \Omega \cap Q_{p}=\left(\xi \in O_{P} \mid \xi_{N}<H_{P}\left(\xi_{1}, \ldots, \xi_{N-1}\right)\right)$ where $Q_{P}$ is a neighborhood of the origin and $F_{P}, C\left(\mathbf{R}^{N-1}\right), F_{p}(0)=0$.

Under this general assumption on $\Omega$ it is not known if a solution of problem $(0.1),(0.2)$ exists and if it exists, the rate of blow-up at the boundary is not known. Therefore, in contrast to previous works, the uniqueness result is not based on information of this type, but on a direct comparison of solutions. More precisely, it is shown that if $u_{1}, u_{2}$ are solutions of the problem, then $u_{1} / u_{2} \rightarrow 1$ at the boundary. ( $\Lambda$ preliminary version of this result was presented in $[\mathrm{MV}])$.

(iii) Rate of blow-up at the boundary. It is known that if $\partial \Omega$ is of class $C^{2}$ then every solution $u$ of problem $(0.1),(0.2)$ (with $k \equiv 1$ ) satisfies,

$$
u(x) \delta(x)^{2 /(p-1)} \rightarrow c_{p} \text { as } \delta(x) \rightarrow 0
$$

where $c_{p}$ is a constant.

We show that this result remains valid if $\Omega$ is of class $C^{1}$. In addition. if $\Omega$ is a domain with piecewice $C^{\perp}$ boundary, we prove that the rate of blow-up is the same as above but the limit in (0.3) exists only if $x \rightarrow P$ where $P$ is a regular point of $\partial \Omega$. Otherwise the limit depends on the direction of approach to $P$ and on the geometry of the boundary near $P$. (For a complete description of the asymptotic behavior of solutions in this case, see Theorem 3.7). Finally, if $\Omega$ is a Lipschitz domain, we show that the rate of blow-up is the same as above, but the limit in (0.3) may not exist. (Theorem 2.5).

If the domain is not Lipschitz the rate of blow-up of solutions at the boundary will in general depend on the limiting point and may be lower than the rate described in (0.3). Such behavior can be observed in the case of domains whose boundary contains a re-entrant cusp, (see Ch. 4). Suppose that $\Omega$ is a domain with smooth boundary except for a standard re-entrant cusp at $P_{0}$. Let $(r, \sigma)$ denote a set of spherical coordinates centered at $P_{0}$. Then, if $1<p<(N-1) /(N-3)$ every solution $u$ of problem $(0.1)$, (0.2) exhibits the following behavior near $P_{0}$ :

$$
u(r, \sigma) r^{2 /(p-1)} \rightarrow w(\sigma) \text { as } r \rightarrow 0,
$$

where $w$ is a solution of a semilinear equation on $S^{N-1}$ (related to $(0.1)$ ) which blows up at one point (essentially the point where the "axis" of the cusp intersects the sphere). However if $p \geq(N-1) /(N-3)$, then $(0.4)$ 
holds with $w=0$ (Theorem 4.1). In this case the rate of blow-up is lower than $r^{-2 /(p-1)}$ depending on the geometry of the cusp. (For details see Theorem 4.2 and 4.6).

The investigation of the behavior of solutions near the boundary depends in an essential way on the following principle which is closely related to the uniqueness result.

(iv) Localization principle. Let $\Omega$ be a domain satisfying the local graph property and let $\Gamma$ be a relatively open subset of its boundary. Let $u_{1}$ and $u_{2}$ be solutions of $(0.1)$ which blow-up as $x \rightarrow \Gamma$. Then $u_{1} / u_{2} \rightarrow 1$ locally uniformly as $x \rightarrow \Gamma$ (Proposition 2.4).

(v) Stability. Suppose that $\Omega$ is a domain satisfying the local graph property with $F_{p}$ Lipschitz (see (ii)). In this case problem $(0.1),(0.2)$ possesses a unique solution which is stable in the following sense.

(a) The solution $u$ depends continuously on $k$. More precisely if $u_{i}$ is a solution of the problem with $k$ replaced by $k_{i}(i=1,2 \ldots)$ and $k_{i} / k \rightarrow 1$ uniformly then $u_{i} / u \rightarrow 1$ uniformly (This is a consequence of Proposition 2.4).

(b) The solution $u$ depends continuously on the domain (See Proposition 2.7).

\section{A COMPARISON LEMMA AND EXISTENCE RESULTS}

Let $\Omega$ be a bounded domain in $\mathbb{R}^{N}, N \geq 2$. In this section we consider positive solutions of the equation,

$$
\Delta u+h u-k|u|^{p-1} u=-f \quad \text { in } \Omega .
$$

where $h, k, f \in C(\bar{\Omega}), k>0, f \geq 0$ and $p>1$. (For technical reasons it will be convenient to assume that $h, k, f$ are defined and continuous in a neighborhood of $\bar{\Omega}$.) A positive solution of (1.1) which satisfies the condition,

$$
u(x) \rightarrow \infty \quad \text { as } \delta(x)=\operatorname{dist}(x, \partial \Omega) \rightarrow 0
$$

will be called a large solution. We start with a comparison result.

Lemma 1.1. - Let $\Omega$ be a bounded domain. Let $u_{1}$ be a weak positive supersolution and $u_{2}$ a weak non-negative subsolution of (1.1) belonging to $W_{\text {loc }}^{1,2}(\Omega) \cap C(\Omega)$. Accordingly, if $w$ is a non-negative function in $\dot{W}^{1,2}(\Omega)$ which has compact support in $\Omega$, then,

$$
\int_{\Omega}\left(\nabla u_{1} \nabla w-h u_{1} w+k u_{1}^{p} w\right) d x \geq \int_{\Omega} f w d x
$$


and

$$
\int_{\Omega}\left(\nabla u_{2} \nabla w-h u_{2} w+k u_{2}^{p} w\right) d x \leq \int_{\Omega} f w d x .
$$

Under these assumptions, if

$$
\limsup _{\delta(x) \rightarrow 0}\left(u_{2}-u_{1}\right)(x) \leq 0, \quad(\operatorname{with} \delta(x)=\operatorname{dist}(x, \partial \Omega)),
$$

then $u_{1} \geq u_{2}$ in $\Omega$. If, in addition, (1.4) holds with strict inequality and $u_{1}, u_{2}$ are in $C^{2}(\Omega)$ then $u_{1}>u_{2}$ in $\Omega$.

Proof. - We shall employ (a variant of) a method due to [BBL]. Let $\varepsilon_{1}>\varepsilon_{2}>0$ and denote $w_{i}=\left(u_{i}+\varepsilon_{i}\right)^{-1}\left(\left(u_{2}+\varepsilon_{2}\right)^{2}-\left(u_{1}+\varepsilon_{1}\right)^{2}\right)_{+}$ $(i=1,2)$. Observe that $w_{i}$ belongs to $W_{\mathrm{loc}}^{1,2}(\Omega)$ and (in view of (1.4)) it has compact support in $\Omega$. Using $(1.3)_{1}$ with $w=w_{i}$ and substracting, we obtain

$$
\begin{aligned}
& -\int_{\Omega}\left[\nabla u_{2} \nabla w_{2}-\nabla u_{1} \nabla w_{1}\right] d x-\int_{\Omega} f\left(w_{1}-w_{2}\right) d x \\
& \geq \int_{\Omega} k(x)\left(u_{2}^{p} w_{2}-u_{1}^{p} w_{1}\right)+\int_{\Omega} h(x)\left(u_{1} w_{1}-u_{2} w_{2}\right)
\end{aligned}
$$

Denote $\Omega_{+}\left(\varepsilon_{1}, \varepsilon_{2}\right)=\left\{x \in \Omega: u_{2}(x)+\varepsilon_{2}>u_{1}(x)+\varepsilon_{1}\right\}$ and note that the integrands in (1.5) vanish outside this set. The first integral on the 1.h. side of (1.5) equals,

$$
-\int_{\Omega_{+}\left(\varepsilon_{1}, \varepsilon_{2}\right)}\left[\left|\nabla u_{2}-\frac{u_{2}+\varepsilon_{2}}{u_{1}+\varepsilon_{1}} \nabla u_{1}\right|^{2}+\left|\nabla u_{1}-\frac{u_{1}+\varepsilon_{1}}{u_{2}+\varepsilon_{2}} \nabla u_{2}\right|^{2}\right] d \sigma .
$$

Noting that $w_{1}>w_{2}$ in $\Omega_{+}\left(\varepsilon_{1}, \varepsilon_{2}\right)$, we conclude that the 1.h. side of (1.5) is not positive. On the other hand as $\varepsilon_{1} \rightarrow 0$ (recall that $\varepsilon_{1}>\varepsilon_{2}>0$ ) the r.h. side of (1.5) converges to

$$
\int_{\Omega_{+}(0,0)} k(x)\left(u_{2}^{p}{ }^{1}-u_{1}^{p}{ }^{1}\right)\left(u_{2}^{2}-u_{1}^{2}\right) d x .
$$

(Indeed, in $\Omega_{+}\left(\varepsilon_{1}, \varepsilon_{2}\right), u_{2}>u_{1}>0$ so that $w_{i} \rightarrow u_{i}^{-1}\left(u_{2}^{2}-u_{1}^{2}\right)$ as $\varepsilon_{1} \rightarrow 0$. In addition the integrands on the r.h. side of (1.5) are dominated by integrable functions.) Unless $\Omega_{+}(0,0)$ is empty, the limiting value of the r.h. side of (1.5) is positive.

Since this leads to a contradiction we conclude that $u_{2} \leq u_{1}$ in $\Omega$. If $u_{1}, u_{2} \in C^{2}(\Omega)$ and (1.4) holds with strict inequality then, by the strong maximum principle, $u_{2}<u_{1}$ in $\Omega$. (Indeed if $u_{1}=u_{2}$ at some point in $\Omega$, 
then there exists an arbitrarily small ball $B \subset \Omega$ such that $u_{1}=u_{2}$ at its center, but $u_{1}$ is not identical to $u_{2}$ in $B$. This contradicts the generalized maximum principle.

Remark 1.2. - The following variant of lemma 1.1 can be established by the same argument as above.

Let $u_{1}$ be a weak positive supersolution and $u_{2}$ a weak non-negative subsolution of (1.1) belonging to $W^{1,2}(\Omega)$. (Accordingly, $(1.3)_{1,2}$ holds whenever $w$ is a non-negative function in $W^{1.2}(\Omega)$.) Under these assumptions, if $\left(u_{2}-u_{1}\right)_{+} \in W^{1,2}(\Omega)$ then $u_{1} \geq u_{2}$ in $\Omega$.

Employing lemma 1.1 , a standard argument yields the following existence result.

Lemma 1.3. - Let $\Omega$ be a bounded domain with $C^{2}$ boundary. Suppose that h. $k . f \in C^{n}(\bar{\Omega})$ for some $\alpha \in(0,1)$. Then there exists a large solution of $(1.1)$ in $C^{2}(\Omega)$.

Proof. - If $\psi$ is a non-negative function belonging to $W^{1,2}(\Omega) \cap C(\bar{\Omega})$, then there exists a bounded, non-negative solution $u$ of $(1.1)$ in $W^{1,2}(\Omega) \cap L^{\infty}(\Omega)$ such that $(u-\psi) \in W^{1,2}(\Omega)$. Indeed, $\underline{u} \equiv 0$ is a subsolution of our problem, while $\bar{u} \equiv M$ where $M$ is a constant such that $M \geq \sup _{\partial \Omega} \psi$ and $k M^{p}-h M \geq f$ in $\Omega$, is a supersolution. Thus there exists a solution $u$ as above, such that $0 \leq u \leq M$. If in addition $\psi \in W^{2, \infty}(\Omega)$ then by classical regularity theory, $u \in C^{2, \alpha}(\Omega) \cap C(\bar{\Omega})$. Finally by the strong maximum principle, $u>0$ in $\Omega$. By the previous lemma this solution is unique.

Now let $u_{n}$ be the solution of (1.1) satisfying $u_{n}=n$ on $\partial \Omega, n=1,2, \ldots$ The estimates of Keller [K] and Osserman [O] imply that $\left\{u_{n}\right\}$ is locally uniformly bounded in $\Omega$. By the previous lemma $\left\{u_{n}\right\}$ is monotone increasing. Consequently, $\left\{u_{n}\right\}$ converges locally uniformly in $\Omega$ to a solution $u$ of (1.1) which obviously satisfies (1.2).

Remarks 1.4. - (i) The solution $u$ obtained as the limit of $\left\{u_{n}\right\}$ is obviously the minimal large solution. It is in fact the supremum of all bounded positive solutions of $(1.1)$ in $\Omega$.

(ii) If $\Omega$ is an arbitrary bounded domain and if there exists a large solution of (1.1) in $\Omega$, then there exists a maximal solution, i.e. a solution which dominates every positive solutions of (1.1) in $\Omega$. Indeed if $\left\{\Omega_{n}\right\}$ is a sequence of smooth subdomains of $\Omega$ such that $\bar{\Omega}_{n} \subset \Omega_{n+1}$ and if $v_{n}$ is a large solution of (1.1) in $\Omega_{n}$, then $\left\{v_{n}\right\}$ is monotone decreasing and converges (locally uniformly in $\Omega$ ) to a solution $v$ of (1.1). Lemma 1.1 implies that $v$ dominates every positive solution of $(1.1)$ in $\Omega$. 
(iii) The result stated in Lemma 1.3 remains valid if $\Omega$ is a domain contained in the unit sphere $S^{N-1}$ and if $\Delta$ is replaced by the LaplaceBeltrami operator $\Delta_{g}$ in (1.1). Indeed, the stereographic projection $\mathbb{P}_{N}$ transforms this problem into a similar problem in the domain $\mathbb{P}_{N} \Omega$ in $\mathbb{R}^{N-1}$.

Next we describe a construction of certain special solutions of (1.1) that will play a key role in the proofs of the existence and uniqueness results to follow.

LEMMA 1.5. - Let $\Omega$ be an arbitrary domain in $\mathbb{R}^{N}$ and suppose that there exists a large solution of the equation $\Delta u=u^{p}$ in $\Omega$. Let $\Xi$ be a compact subset of $\partial \Omega$ and let $P \in \Xi$. Suppose that, for every $\delta>0$, there exists an open, connected neighborhood of $P$, say $Q_{P}$, with $C^{2}$ boundary, such that,

$\Omega_{P}=Q_{P} \cap \Omega$ is a simply connected domain,

$Q_{P} \subset \Xi_{\delta}=\{x: \operatorname{dist}(x, \Xi)<\delta\}$ and $\partial \Omega \cap \bar{\Omega}_{P}=\overline{\partial \Omega \cap Q_{P}}$.

Then there exists $\delta_{0}>0$ (which depends on $\Xi$ but not on $P$ ) such that, if $\Omega_{P}$ is contained in $\Xi_{\delta_{1}}$, the following statements hold.

(a) There exists a large solution of (1.1) in $\Omega_{P}$;

(b) There exists a positive solution $v$ of (1.1) in $\Omega_{P}$ such that,

$$
\begin{aligned}
& v(x) \rightarrow \infty \text { locally uniformly as } x \rightarrow \Gamma_{1}=\partial \Omega \cap Q_{P}, \\
& v \in C\left(\Omega_{P} \cup \Gamma_{2}\right) \quad \text { and } \quad v=0 \text { on } \Gamma_{2}=\Omega \cap \partial Q_{P} .
\end{aligned}
$$

Proof. $-(a)$ Let $b=2 \sup _{\Omega} k$ and let $c=\sup \left\{-h(x) t-\frac{1}{2} b t^{p}: t>0\right.$, $x \in \Omega\}$. Then, every positive solution $u$ of (1.1) satisfies

$$
\Delta u \leq b u^{p}+c
$$

Let $U$ be a large solution of $\Delta u=2 b u^{p}$ in $\Omega$. Let $M=\inf \{U(x): x \in$ $\left.\Omega \cap \Xi_{\delta}\right\}$ and choose $\delta_{0}$ sufficiently small so that $b M^{p} \geq c$. Then

$$
\Delta U \geq b U^{p}+c \text { in } \Omega_{P} .
$$

Let $\left\{\Theta_{n}\right\}$ be an increasing sequence of domains with $C^{2}$ boundary such that

$$
\bar{\Theta}_{n} \subset \Theta_{n+1} \subset \Omega_{P} \quad \text { and } \quad \Theta_{n} \uparrow \Omega_{P} .
$$

Let $u_{n}$ and $V$ be large solutions of (1.1) in $\Theta_{n}$ and $Q_{P}$ respectively. By lemma $1.1\left\{u_{n}\right\}$ is monotone decreasing and $u_{n} \geq V$ in $\Theta_{n}$. By the maximum principle, (1.7) and (1.8) $u_{n} \geq U$ in $\Theta_{n}$. Hence $\lim u_{n}$ is a large solution of $(1.1)$ in $\Omega_{P}$. 
(b) In the proof of the second statement we may assume (in view of $(a)$ ) that there exists a large solution of (1.1) in $\Omega$. Now, let $\left\{\Theta_{n}\right\}$ be an increasing sequence of domains with $C^{2}$ boundary such that,

$\Theta_{n} \subset \Omega_{P}, \Theta_{n} \uparrow \Omega_{P}$ and $\Omega_{P} \backslash \Theta_{n} \subset K_{n}=\left\{x: \operatorname{dist}\left(x, \Gamma_{1}\right)<2^{-n}\right\}$.

Denote $\Gamma_{1, n}=\partial \Theta_{n} \cap K_{n}, \Gamma_{2, n}=\partial \Theta_{n} \cap\left(\bar{K}_{n}\right)^{c}$. Thus $\Gamma_{2, n} \subset \Gamma_{2, n+1} \subset \Gamma_{2}$. We shall also assume that the sets $\Gamma_{1, n}$ are disjoint.

For every $n$, consider a sequence of functions $\left\{\varphi_{n, k}\right\}_{k=1}^{\infty}$ on $\partial \Theta_{n}$ satisfying the following properties.

$\varphi_{n, k}=k$ on $\Gamma_{1, n} ; \varphi_{n, k}=0$ for $x \in \Gamma_{2, n}$ such that dist $\left(x, \Gamma_{1, n}\right)>2^{-n}$;

$0 \leq \varphi_{n, k} \leq k$ everywhere; $\varphi_{n, k} \in C^{2}\left(\partial \Theta_{n}\right)$;

$\varphi_{n, k} \geq \varphi_{n-1, k}$ on $\Gamma_{2, n}$ and $\varphi_{n, k} \leq \varphi_{n, k-1}$ on $\partial \Theta_{n}$.

Let $v_{n, k}$ be a solution of (1.1) in $\Theta_{n}$ such $v_{n, k}=\varphi_{n, k}$ on $\partial \Theta_{n}$. By lemma $1.1\left\{v_{n, k}\right\}_{k=1}^{\infty}$ is monotone increasing and (by a standard argument) the sequence is locally bounded. Hence $v_{n}=\lim _{k \rightarrow \infty} v_{n, k}$ is a solution of (1.1) in $\Theta_{n}$ such that

and

$$
\left.\begin{array}{l}
v_{n} \rightarrow \infty \quad \text { as } x \rightarrow \Gamma_{1, n} ; v_{n} \in C\left(\Theta_{n} \cup \Gamma_{2, n}\right) \\
v_{n}=0 \text { on } \Gamma_{2, n} .
\end{array}\right\}
$$

Furthermore, by their construction, $v_{n, k} \geq v_{n+1, k}$ so that $\left\{v_{n}\right\}$ is monotone decreasing. Consequently $v=\lim _{n \rightarrow \infty} v_{n}$ is a solution of (1.1) in $\Omega_{P}$. If $V$ is a large solution of (1.1) in $Q_{P}, v_{n}+V$ is a supersolution of (1.1) in $\Theta_{n}$ which blows up on $\partial \Theta_{n}$. Hence $v_{n}+V \geq U$, where $U$ is a large solution of (1.1) in $\Omega$. Thus $v \mid V \geq U$ and this implies (1.6) $)_{1}$. Finally, by (1.9) $v$ satisfies $(1.6)_{2}$.

Remark 1.6. - (i) If $\Omega$ is bounded and if the assumptions of lemma 1.5 holds w.r. to every $P \in \partial \Omega$ then the existence of a large solution of the equation $\Delta u=u^{p}$ in $\Omega$ implies the existence of a large solution of (1.1) in $\Omega$ and conversely.

The first statement follows from part $(b)$ of the lemma. Consider a sequence of smooth domains $\left\{\Omega_{n}\right\}$ such that $\Omega_{n} \uparrow \Omega$. If $u_{n}$ is a large solution of (1.1) in $\Omega_{n}$ then $\left\{u_{n}\right\}$ is decreasing and for every $P \in \partial \Omega$, the solution $v$ constructed in part $(b)$ is a subsolution in $\Omega_{n} \cap \Omega_{P}$. Thus $\lim u_{n} \geq v$ in $\Omega_{P}$.

An examination of the proof shows that the lemma remains valid if the roles of the two equations involved in it are reversed. Therefore the same is true w.r. to the statement of the remark. 
(ii) If it is assumed that there exists a large solution of (1.1) in $\Omega$ then the assumption " $Q_{P} \subset \Xi_{\delta}$ " is not needed for statement $(b)$. (In fact it is even sufficient to assume that there exists a local subsolution of (1.1) near the boundary, which blows up on $\partial \Omega$.)

Using the construction described in the previous lemma we can extend the existence result of lemma 1.3 to a larger class of domains as defined below.

Definition 1.7. - A domain $\Omega$ satisfies the exterior cone condition if there exists a bounded spherical cone $K$ (e.g. $K=\{(r, \sigma): 0<r<R, \sigma \in S\}$ where $S$ is a spherical cap on $S^{N-1}$ ), such that for every $P \in \partial \Omega$ there exists acone $K_{P}$ with vertex $P$ and congruent to $K$, which is contained in the complement of $\Omega$. The transformation mapping $K$ onto $K_{P}$ (composed of a rotation followed by a translation) will be denoted by $T_{P}$.

A domain $\Omega$ satisfies the exterior segment condition if the previous condition holds, with $K$ a bounded segment.

PROPOSITION 1.8. - If $\Omega$ is a bounded domain in $\mathbb{R}^{N}$ satisfying the exterior cone condition, Equation (1.1) possesses at least one large solution. Furthermore, there exists a function $\varphi \in C(0, \infty)$, which tends to infinity at zero, and a positive number $\delta_{0}$ (both of which depend only on $K$ ), such that every large solution $u$ of (1.1) satisfies,

$$
\begin{gathered}
u(x) \geq \varphi(\operatorname{dist}(x, \partial \Omega)), \quad \text { in }\left\{x \in \Omega: \operatorname{dist}(x, \partial \Omega) \leq \delta_{0}\right\} . \\
\text { Proof. - Consider a cone } C_{S}^{R}=\{(r, \sigma): 0<r<R, \sigma \in S\} . \text { Denote, } \\
\Gamma_{1}=\{(r, \sigma): 0 \leq r<R, \sigma \in \partial S\}, \quad \Gamma_{2}=\{(r, \sigma): r=R, \sigma \in S\} .
\end{gathered}
$$

We claim that for $\Omega=C_{S}^{R}$ there exists a solution of (1.1) such that,

$$
\begin{gathered}
u \in C^{2}\left(C_{S}^{R}\right) \cap C\left(C_{S}^{R} \cup \Gamma_{2}\right) \quad \text { and } \quad u=0 \text { on } \Gamma_{2}, \\
u(x) \rightarrow \infty \text { locally uniformly as } x \rightarrow \Gamma_{1} .
\end{gathered}
$$

This is a consequence of lemma 1.5 and the fact that there exists a large solution of the equation $\Delta u=u^{p}$ in the unbounded cone $C_{S}=C_{S}^{\infty}$. Such a solution is given by,

$$
u_{S}(r, \sigma)=r^{-2 /(p-1)} w_{S}(\sigma)
$$

Vol. 14, n 2-1997. 
where $w_{S}$ is a large solution in $S$ of the equation,

$$
\left.\begin{array}{c}
\Delta_{g} w+\lambda(N, p) w-w^{p}=0 \\
\left.\lambda(N \cdot p)=\frac{2}{p-1}\left(\frac{2 p}{p-1}-N\right)\right] \cdot
\end{array}\right\}
$$

Now suppose that the cone $K$ mentioned in Definition 1.7 is given by $K=C_{S}^{R}$ and let $K^{\prime}$ denote its complement in the $R$-ball. Let "? be a positive solution (in $K^{\prime}$ ) of the equation,

$$
\Delta \eta=\bar{k} v^{\prime \prime}-\underline{h} v \quad\left(\bar{k}=\sup _{\underline{l}} k, \underline{h}=\inf _{\Omega} h\right)
$$

which satisfies the boundary conditions corresponding to $(1.11)_{1,2}$. Thus $v$ vanishes on the spherical boundary of $K^{\prime}$ and blows up (locally uniformly) on the lateral boundary. Finally let $K_{P}^{\prime}=T_{P} K^{\prime}$ and $v_{P}=v^{\prime} \circ T_{P}$.

Let $\left\{\Omega_{n}\right\}$ be a sequence of domains with smooth boundary such that $\bar{\Omega}_{n} \subset \Omega_{n+1} \subset \Omega$ and $\Omega_{n} \uparrow \Omega$. Let $u_{n}$ be a large solution of (1.1) in $\Omega_{n}$. By lemma $1.1\left\{u_{n}\right\}$ is decreasing and $u_{n} \geq v_{P}$, in $K_{P}^{\prime} \cap \Omega_{n}$. This implies the stated result.

Remark 1.9. - If $3<N$ and $1<p<(N-1) /(N-3)$ then Proposition 1.8 remains valid if the exterior cone condition is replaced by the (weaker) exterior segment condition. Similarly if $1<p<$ $(N-k-1) /(N-k-3)$ for some integer $k$ such that $0 \leq k \leq N-1$ then the proposition remains valid if the exterior cone condition is replaced by the exterior $(k+1)$-dimensional plane condition.

We sketch the proof which is similar to that of Proposition 1.8. If $k:=0$ Equation (1.13) possesses a positive solution in $S^{N-1}$ which blows up at one point, say $\sigma$. Let $w_{S}, S=S^{N-1} \backslash\left\{\sigma_{0}\right\}$, be such a solution and let $u_{S}$ be as in (1.12). Then $u_{S}$ satisfies the equation $\Delta u=u^{\prime \prime}$ in $D_{\sigma_{\|}}=\left\{(r, \sigma): 0<r, \sigma \in S^{x-1}, \sigma \neq \sigma_{0}\right\}$ and blows up at the boundary. Using this fact one can proceed as in the proof of Proposition 1.8.

For $0 \leq k \leq N-4$, if $p \geq(N-k-1) /(N-k-3)$, singularities of solutions of (1.13) concentrated on a $k$-dimensional submanifold of $S^{N-1}$ are removable (see [V2]). However, if $1<p<(N-k-1) /(N-k-3)$, there exist solutions of (1.13) which are singular on a $k$-dimensional submanifold. Therefore there exist solutions of $\Delta u=u^{p}$ of the form (1.12) with $S=S^{N-1} \backslash \Lambda$ where $\Lambda$ is a $k$-dimensional submanifold of $S^{N-1}$. Consequently, for such values of $p$, the existence of large solutions can be established if the domain satisfies an exterior $(k+1)$-dimensional plane condition. 
COROLlaRY 1.10. - if $\Omega$ is a bounded domain in $\mathbb{R}^{N}$ satisfying the exterior cone condition, equation (1.1) possesses a minimal and a maximal large solution.

Proof. - The large solution constructed in the proof of the proposition is obviously the maximal large solution. In order to obtain the minimal large solution we consider a sequence of domains $\left\{G_{n}\right\}$ such that $\Omega \subset \bar{G}_{n+1} \subset G_{n}, \cap G_{\boldsymbol{I}_{n}}=\Omega$ and $\left\{G_{n}\right\}$ satisfies the exterior cone condition uniformly, i.e. there exists a cone $K$ as in Definition 1.7 such that, for every $n, G_{n}$, satisfies the exterior cone condition with respect to $K$. Let $w_{n}$ be a large solution in $G_{n}$. Then $\left\{w_{n}\right\}$ is monotone increasing in $\Omega$ and it converges to a solution $w$ of (1.1). The last statement of Proposition 1.8 implies that the rate of blow up of $w_{n}$ at $\partial G_{n}$ is uniform with respect to $n$. Since $w_{n}<w, w$ blows up at $\partial G$. Clearly $w$ is the minimal large solution.

\section{UNIQUENESS, BOUNDARY ESTIMATES AND CONTINUOUS DEPENDENCE OF LARGE SOLUTIONS ON THE DOMAIN}

In this section we present an uniqueness result for large solutions of (1.1) in domains with non-smooth boundary, we derive boundary estimates for such solutions in Lipschitz domains and we demonstrate their continuous dependence on the domain. We start with a definition needed for the statement of our uniqueness result.

Definition 2.1. - Let $\Omega$ be a domain in $\mathbb{R}^{N}$. We shall say that $\Omega$ satisfies the local graph property if for every boundary point $P$ there exist a neighborhood $Q_{P}$, a set of coordinates $\xi$ obtained from $x$ by rotation and a function $F_{P} \in C\left(\mathbb{R}^{N-1}\right)$ such that

$$
Q_{P} \cap \cap \Omega=Q_{P} \cap G\left(F_{P}\right) \text { with } G\left(F_{P}\right)=\left\{\xi: \xi_{N}<F_{P}\left(\xi_{1}, \ldots, \xi_{N-1}\right)\right\} .
$$

The class of domains $\Omega$ satisfying this property will be denoted by $C_{g r}$. If this condition holds with $F_{P} \in C^{0.1}\left(\mathbb{R}^{N-1}\right)$ we shall say that $\Omega$ is of class $C_{g r}^{0.1}$.

Note that every bounded domain of class $C_{g r}$ (resp. $C_{g r}^{0,1}$ ) possesses the exterior segment (resp. cone) condition. In fact it is known that the local graph property is equivalent to the segment property, but this fact will not be used here.

The following is our main uniqueness result. A special case, dealing with the equation $\Delta u=u^{p}$, was proved in [MV].

Vol. 14, $n^{3} 2-1997$ 
ThEOREM 2.2. - Let $\Omega$ be a bounded domain of class $C_{g r}$. Then Problem (1.1) possesses at most one large solution.

Proof. - Suppose that $u$ is a large solution of (1.1). Note that for every $\varepsilon>0$ there exists $\beta_{\varepsilon}>0$ such that

$$
k(1-\varepsilon) u^{p} \leq \Delta u \leq k \cdot(1+\varepsilon) u^{p} \quad \text { in }\left\{x \in \Omega: \operatorname{dist}(x, \partial \Omega)>\beta_{\varepsilon}\right\} .
$$

Let $P \in \partial \Omega$ and assume (as we may) that the set $Q_{P}$ mentioned above is an open, bounded spherical cylinder centered at $P$, with axis parallel to the $\xi_{N}$ axis. Thus,

$$
Q_{P}=\left\{\eta:\left|\eta^{\prime}\right|<\rho_{P},\left|\eta_{N}\right|<\tau_{P}\right\},
$$

where $\eta=\xi-P$ and $\eta^{\prime}=\left(\eta_{1}, \ldots, \eta_{N-1}\right)$. By appropriately choosing $\sigma_{P}$ and $\tau_{P}$ we may also assume that $\partial \Omega$ is bounded away from the 'top' and 'botton' of the cylinder $Q_{P}$ and that $\partial \Omega \cap \bar{Q}_{P}=\overline{\partial \Omega \cap Q_{P}}$. Finally we assume that $\rho_{P}$, and $\tau_{P}$, are sufficiently small so that Lemma 1.5 can be applied to $Q_{P}$ and so that,

$$
\left.\begin{array}{c}
k(P)(1-\varepsilon) u^{\prime}(x) \leq \Delta u \leq h(P)(1+\varepsilon) u^{p}(x), \\
\forall x \in \Theta=Q_{P} \cap \Omega .
\end{array}\right\}
$$

Recall that, by Remark 1.6, the existence of a large solution of (1.1) in $\Omega$ implies the existence of a large solution of the equation $\Delta u=u^{p}$ in $\Omega$. Therefore by Lemma 1.5, there exists a solution $v$ of the problem,

$$
\begin{gathered}
\Delta v=v^{\prime \prime} \text { and } \quad v>0 \text { in } \Theta=Q_{P} \cap \Omega, \\
v(x) \rightarrow \infty \text { locally uniformly as } x \rightarrow Q_{P} \cap \partial \Omega, \\
v(x) \rightarrow 0 \text { locally uniformly as } x \rightarrow \partial Q_{P} \cap \Omega .
\end{gathered}
$$

Next denote,

$v_{1}=(k(P)(1-\varepsilon))^{-1 /(p-1)} v \quad$ and $\quad v_{2}=(k(P)(1+\varepsilon))^{-1 /(p-1)} v$.

and let $w$ be the large solution of equation (2.2) in $Q_{P}$. We claim that,

$$
v_{2}<u<v_{1}+w \text { in } \Theta \text {. }
$$

To verify this claim, let $\xi$ denote the unit vector parallel to the axis of $Q_{P}$ such that $P+\xi$ is outside $\Omega$ and set $\Theta_{\sigma}=\{x-\sigma \xi: x \in \Theta, \sigma>0\}$. If $f$ is a function defined in $\Theta$, set $f_{\sigma}(x)=f(x+\sigma \xi)$ for $x \in \Theta_{\sigma}$. Assume that $\sigma$ is a sufficiently small positive number so that $\Theta_{\sigma} \subset \subset \Omega$. Then $v_{1, \sigma}+w_{\sigma}$ is a supersolution in $\Theta_{\sigma}$ and hence $v_{1, \sigma}+w_{\sigma}>u$ there. On the other hand, by $(2.1), v_{2,-\sigma}<u$ on $\partial\left(\Theta_{-\sigma} \cap \Omega\right)$ and hence $v_{2,-\sigma}<u$ in 
$\Theta_{-\sigma} \cap \Omega$. Thus, for $0<\sigma$ sufficiently small, $v_{2,-\sigma}<u<u_{1, \sigma}+w_{\sigma}$ in $\Theta_{-\sigma} \cap \Theta_{\sigma}$ and hence, letting $\sigma$ tend to zero, we obtain (2.4). Finally, since $w$ is bounded in every compact subset of $Q_{P}$, it follows that

$$
\begin{aligned}
& u(x) /\left(k(x)^{-1 /(p-1)} v(x)\right) \rightarrow 1 \\
& \quad \text { locally uniformly as } x \rightarrow Q_{P} \cap \partial \Omega .
\end{aligned}
$$

Therefore if $u_{1}, u_{2}$ are large solutions of (1.1), then

$$
u_{1}(x) / u_{2}(x) \rightarrow 1 \quad \text { as } \operatorname{dist}(x, \partial \Omega) \rightarrow 0 .
$$

Consequently, for every $\varepsilon>0$ there exists a neighborhood $(\partial \Omega)_{\varepsilon}$ of the boundary where $u_{1} \leq(1+\varepsilon) u_{2}$. Hence, by the maximum principle, the inequality holds in $\Omega$. Thus $u_{1} \leq u_{2}$ and by symmetry, $u_{1}=u_{2}$ in $\Omega$.

COROLlary 2.3. - If $\Omega$ is a bounded domain of class $C_{g r}$ satisfying the exterior cone condition, then Equation (1.1) possesses a unique large solution.

Proof. - This is a consequence of Proposition 1.8 and Theroem 2.2.

We observe that the arguments employed in the proof of the theorem yield also the following.

Proposition 2.4. - Let $\Omega$ be a domain of class $C_{g r}$ (not necessarily bounded).

(i) If $u$ is a large solution of (1.1) and $U$ is a large solution of $\Delta U=U^{p}$ in $\Omega$, then

$$
u(x) /\left(k(x)^{-1 /(p-1)} U(x)\right) \rightarrow 1 \quad \text { locally uniformly as } x \rightarrow \partial \Omega .
$$

(ii) Let $\Gamma$ be a relatively open subset of $\partial \Omega$. Suppose that $\tilde{u}$ is a positive solution of (1.1) such that,

$$
\tilde{u}(x) \rightarrow \infty \quad \text { locally uniformly as } x \rightarrow \Gamma \text {. }
$$

If $u$ is a large solution of $(1.1)$, then

$$
u(x) / \tilde{u}(x) \rightarrow 1 \quad \text { locally uniformly as } x \rightarrow \Gamma .
$$

Proof. - Under the assumptions of part (ii), statement (2.5) holds for every point $P \in \Gamma$, for both $u$ and $\tilde{u}$. Hence (2.9).

Under the assumptions of part (i), $u$ satisfies (2.5) as stated while $U$ satisfies (2.5) with the same function $v$ but with $k=1$. Hence (2.7).

The next result provides estimates near the boundary for large solutions in Lipschitz domains. 
THEOREM 2.5. - Let $\Omega$ be a bounded Lipschitz domain. Then there exist positive constants $c_{1}, c_{2}$ such that the large solution of $(1.1)$ in $\Omega$ satisfies,

$$
c_{1} \delta(x)^{-2 /(p-1)} \leq u(x) \leq c_{2} \delta(x)^{-2 /(p-1)}, \quad \forall x \in \Omega .
$$

where $\delta(x)=\operatorname{dist}(x . \partial \Omega)$.

Proof. - We shall use the notation of Definition 1.7 and Proposition 1.8. First we observe that a bounded Lipschitz domain satisfies both the exterior and the interior cone condition. (The interior cone condition is defined as in Definition 1.7 with the obvious moditication.) One may assume that the same basic cone $K=C_{S}^{R}$ is associated with both conditions. For $P \in \partial \Omega$ let $K_{P}$ be as in Definition 1.7 and let $\hat{K}_{P}$ be its counterpart with respect to the interior cone condition. Since $\Omega$ is Lipschitz we may assume that $K_{\Gamma}$ and $\hat{K}_{P}$ vary continuously with $P$. Finally let $K_{P}^{\prime}$, denote the complement of $K_{P}$ in the $K$-ball centered at $I^{\prime}$.

Let $v$ and $v_{P}$ be solutions of (1.14) in $K^{\prime}$ and $K_{P}^{\prime}$ as in the proof of Proposition 1.8. Similarly let $w$ be a large solution of the equation

$$
\Delta w=\underline{k} w^{p}-\bar{h} w-\bar{f} \quad\left(\underline{k}=\inf _{\Omega} h, \bar{h}=\sup _{\Omega} h, \bar{f}=\sup _{\Omega} f\right)
$$

in $K$ and let $w_{P}$ be the corresponding solution in $\hat{K}_{P}$. Assuming that $K=C_{S}^{R}$ let $S^{\prime}=S^{N-1} \backslash S$ (so that $\left.K^{\prime}=S_{S^{\prime}}(R)\right)$ and let $u_{S}$ and $u_{S^{\prime}}$ be as in (1.12). By Proposition 2.4 (ii),

$$
\begin{aligned}
& u(x) /\left(\underline{k}^{-1 /(p-1)} u_{S}(x)\right) \rightarrow 1 \text { locally uniformly as } x \rightarrow \Gamma_{1}, \\
& v(x) /\left(\bar{k}^{-1 /(p-1)} u_{S^{\prime}}(x)\right) \rightarrow 1 \text { locally uniformly as } x \rightarrow \Gamma_{1}^{\prime},
\end{aligned}
$$

where $\left.\Gamma_{1}=\{(r, \sigma): 0 \leq r<R, \sigma \in \partial S\}\right)$ and $\Gamma_{1}^{\prime}=\{(r, \sigma): 0 \leq$ $\left.r<R, \sigma \in \partial S^{\prime}\right\}$. From these relations and (1.12) we conclude that if $S_{0}$ and $S_{0}^{\prime}$ are compact subsets of $S$ and $S^{\prime}$ respectively, there exist positive constants $b_{0}, b_{1}, b_{0}^{\prime}, b_{1}^{\prime}$ such that

$$
\begin{aligned}
& b_{0}^{\prime} \leq v(x) / r^{-2 /(p-1)} \leq b_{1}^{\prime}, \quad x \in C_{S_{0}^{\prime}}(R / 2) \\
& b_{0} \leq w(x) / r^{-2 /(p-1)} \leq b_{1}, \quad x \in C_{S_{0}}(R / 2) .
\end{aligned}
$$

Now if $u$ is the large solution of (1.1) in $\Omega$ then for every $P \in \partial \Omega$,

$$
v_{P} \leq u \text { in } K_{P^{\prime}} \cap \Omega, \quad u \leq w_{P} \text { in } \hat{K}_{P} .
$$

These inequalities together with $(2.13)_{1.2}$ imply $(2.10)$. 
For the statement of the next result we need an additional definition.

Definition 2.6. - If $A, B$ are two bounded sets in $\mathbb{R}^{N}$ we denote,

and

$$
\left.\begin{array}{l}
\delta_{A}(B)=\sup \{\operatorname{dist}(x, A): x \in B\} \\
\delta(A, B)=\delta_{A}(B)+\delta_{B}(A) .
\end{array}\right\}
$$

If $\left\{A_{n}\right\}$ is a sequence of bounded open sets and $B$ is a bounded open set. we say that $\left\{A_{n}\right\}$ converges to $B$ if $\delta\left(A_{n}, B\right) \rightarrow 0$ and $\delta\left(\partial A_{n}, \partial B\right) \rightarrow 0$.

PROPOSITION 2.7. - Let $\Omega$ be a bounded domain of class ar. Le: $\left\{D_{n}\right\}$ be a sequence of bounded domains of class $C_{g r}^{0,1}$ such that $D_{n} \rightarrow \Omega$. Let $v_{n}$ and $u$ denote the large solutions of $(1.1)$ in $D_{n}$ and $\Omega$ respectively. Then $v_{n} \rightarrow u$ locally uniformly in $\Omega$.

Proof - Let $\left\{\Omega_{n}\right\}$ be an increasing sequence of subdomains of $\Omega$ as in Proposition 1.8 and let $\left\{G_{n}\right\}$ be a decreasing sequence of domains as in Corollary 1.10. If $u_{n}$ (resp. $w_{n}$ ) is a large solution of $(1.1)$ in $\Omega_{n}$ (resp. $\left.G_{n}\right)$ and $u$ is the unique large solution of $(1.1)$ in $\Omega$ then both $\left\{u_{n}\right\}$ and $\left\{w_{n}\right\}$ converge to $u$ locally uniformly in $\Omega$ (see proof of 1.8 and 1.10 ). Let $v_{j}$ denote the large solution of (1.1) in $D_{j}$. For every $n$ there exists $j_{n}$ such that $\Omega_{n} \subset D_{j_{n}} \subset G_{n}$. Hence there is a subsequence of $\left\{v_{j}\right\}$ which converges to $u$ locally uniformly in $\Omega$. Since the limit is independent of the subsequence, it follows that $v_{j} \rightarrow u$ locally uniformly in $\Omega$.

Remark 2.8. - The results presented in this section, remain valid for Equation (1.1), with $\Delta$ replaced by the Laplace-Beltrami operator $\Delta_{q}$, in domains on the unit sphere $S^{N-1}$. Indeed, the stereographic projection transforms Equation (1.1) in a domain $S$ in $S^{N-1}$ into an equation of the same type in a domain $S^{*}$ in $\mathbb{R}^{N-1}$ which is the image of $S$ by this projection. In this connection, we shall say that $S$ is of class $C_{g r}$ or that $S$ satisfies the exterior cone condition if $S^{*}$ has this property.

\section{ASYMPTOTIC BEHAVIOR AT CORNERS AND EDGES}

In the previous section we obtained the rate of blow up at the boundary, for large solutions of (1.1) in bounded Lipschitz domains. In this section we describe the precise asymptotic behavior of such solutions in domains whose boundary is piecewise $C^{1}$.

For $0 \leq k \leq N-2$, let $\Pi_{k}$ be a $k$-dimensional subspace of $\mathbb{R}^{N}$, say $\left\{x \in \mathbb{R}^{N}: x_{j}=0, j=1, \ldots, N-k\right\}$. For $k=0$ we assume that $\Pi_{k}$ is a 
point, say the origin. Denote by $(r, \sigma, z)$ a set of cylindrical coordinates in $\mathbb{R}^{N}$ with axis $\Pi_{k}$, e.g. $z=\left(z_{1}, \ldots, z_{k}\right)$ with $z_{i}=x_{N-k+i}$ and $(r, \sigma)$ polar coordinates in $\Pi_{k}^{\prime}$ (=the orthogonal complement of $\Pi_{k}$ ). Given a domain $S$ in $S^{N-k-1}$ (the unit sphere in $R^{N-k}$ ) we denote by $W_{S}\left(\Pi_{k}\right)$ the cone (or wedge),

$$
W_{S}\left(\Pi_{k}\right)=\left\{(r, \sigma, z): 0<r, \sigma \in S, z \in R^{k}\right\}
$$

and by $W_{S}^{R}\left(\Pi_{k}\right)$ the intersection of $W_{S}\left(\Pi_{k}\right)$ with $B^{R}$ (= the ball of radius $R$ centered at the origin in $\left.\mathbb{R}^{N}\right) . \Pi_{k}$ will be called the edge of $W_{S}\left(\Pi_{k}\right)$.

If $k=0$ and $\Pi_{0}$ is the point $P$ then $W_{S}\left(\Pi_{0}\right)$ (resp. $\left.W_{S}^{R}\left(\Pi_{0}\right)\right)$ is a cone with vertex $P$ which will also be denoted by $C_{S}(P)$ (resp. $C_{S}^{R}(P)$ ). Then we have the following result.

THEOREM 3.1. - Let $S$ be a subdomain of $S^{N-k-1}$ of class $C_{g r}^{0,1}$. Then there exists a unique large solution of the equation $\Delta u=u^{p}$ in $W_{S}\left(\Pi_{k}\right)$. This solution is given by,

$$
u(r, \sigma, z)=r^{-2 /(p-1)} w(\sigma) \text { in } W_{S}\left(\Pi_{k}\right),
$$

where $w$ is the unique large solution of the equation,

$$
\Delta_{g} w+\lambda(N-k, p) w-w^{p}=0 \quad \text { and } \quad w>0 \quad \text { in } S
$$

and $\lambda(m, p)=\frac{2}{p-1}\left(\frac{2 p}{p-1}-m\right)$.

Proof. - The existence and uniqueness of the large solution of (3.3) follows from Proposition 1.8 and Theorem 2.2. A direct computation shows that (3.2) is a solution of the equation $\Delta u=u^{p}$. Thus it remains to shows that the function $u$ given by (3.2) is the unique large solution.

Let $U$ be a large solution of $\Delta u=u^{p}$ in $W_{S}\left(\Pi_{k}\right)$. (Here we only assume that $U$ blows up locally uniformly at the boundary.) Let $v_{1, R}$ (resp. $v_{2, R}$ ) be the unique large solution of this equation in $W_{S}^{R}\left(\Pi_{k}\right)$ (resp. $\Omega=B^{R}$ ). Then $v_{1, R}$ is monotone decreasing with respect to $R$ and $v_{1, R} \geq U$ in $W_{S}^{R}\left(\Pi_{k}\right)$. Consequently $V=\lim _{R \rightarrow \infty} v_{1, R}$ is a large solution of (1.1) in $W_{S}\left(\Pi_{k}\right)$ and $V \geq U$. On the other hand $U+v_{2, R}$ is a supersolution in $W_{S}^{R}\left(\Pi_{k}\right)$, so that $v_{1, R} \leq U+v_{2, R}$. Since $\lim _{R \rightarrow \infty} v_{2, R}=0$ we conclude that $U=V$.

Definition 3.2. - (i) Let $\Omega$ be a domain in $\mathbb{R}^{N}$ and let $\Gamma$ be a $k$ dimensional submanifold of $\partial \Omega$. We shall say that $\Gamma$ is a curved edge of dimension $k$, if for every $P \in \mathrm{I}$ ' there exists an open neighborhood $M$ of $P$ and a $C^{2}$ transformation of coordinates $T$ defined in $M$, such that $T(M \cap \Omega)=T(M) \cap W_{S}\left(\Pi_{k}\right)$ where $S$ is a domain in $S^{N-k-1}$ and $T(\Gamma \cap M) \subset \Pi_{k}$. If $k=0$ and $\Gamma=\{P\}$, we shall say that $P$ is a corner. 
If $\Omega$ is a domain on $S^{N-1}$ a curved edge on $\partial \Omega$ is defined in the same way, but now the range of $T$ is in $\mathbb{R}^{N-1}$.

(ii) If $\Omega$ is a bounded domain in $\mathbb{R}^{2}$ (or in $S^{2}$ ) we shall say that it is piecewise $C^{1}$ if for every $P \in \partial \Omega$, either $P$ is a corner or $\partial \Omega$ is $C^{1}$ in a neighborhood of $P$. If $\Omega$ is a bounded domain in $\mathbb{R}^{N}$ (or in $S^{N}$ ), $N>2$, we define the notion of piecewise $C^{1}$ inductively as follows. First, we say that a curved edge or a corner is piecewise $C^{1}$ if the domain $S$ mentioned in (i) is piecewise $C^{1}$. Then we say that $\Omega$ is piecewise $C^{1}$ if, for every $P \in \partial \Omega$, either $P$ lies on a piecewise $C^{1}$ curved edge of dimension $k$, $0 \leq k \leq N-2$, or $\partial \Omega$ is $C^{1}$ in a neighborhood of $P$.

Note that every bounded domain which is piecewise $C^{1}$ is of class $C_{g r}^{0,1}$.

(iii) If $P \in \partial \Omega$ lies on a curved edge of dimension $h$, we associate with it a limiting wedge $W_{S^{p}}\left(\Pi_{k}\right)$ which is defined as follows. Assume that $P$ is the origin and denote $\Omega_{p}=\Omega \cap B^{\rho}$. Then the family of domains $\Xi_{\rho}=\frac{1}{\rho} \Omega$, converges (as $\rho \rightarrow 0$ ) to a domain $\Xi^{p} \subset B_{1}$ which, in an appropriate set of local coordinates, can be represented in the form $W_{S p}\left(\Pi_{k}\right) \cap B_{1}$. (Here the convergence of $\Xi_{\rho}$ is understood in the sense of Definition 2.6). This notation applies also to points $P$ where $\partial \Omega$ is smooth. Indeed, if a tangent plane exists at $P$ then $k=0$ and $S^{P}$ is a half sphere so that the limiting 'wedge' at $P$ is a half space.

The limiting wedge $W_{S^{P}}\left(\Pi_{k}\right)$ can also be described as follows. Let $T^{\prime}(P)$ be the derivative of the transformation $T$ at $P$. Then

$$
W_{S^{\prime}}\left(\Pi_{k}\right)=T^{\prime}(P)^{-1} W_{S}\left(\Pi_{k}\right) \text {. }
$$

Note that if the curved edge is piecewise $C^{1}$ then $S^{P}$ is piecewise $C^{1}$. Furthermore $S^{P}$ satisfies the properties of class $C_{g r}^{0,1}$ uniformly with respect to $P$ in compact subsets of the curved edge.

Lemma 3.3. - Let $\Omega$ be a domain in $\mathbb{R}^{N}$ and let $P_{0} \in \partial \Omega$. Assuming that $P_{0}$ is the origin, suppose that there exists a ball $B^{R}$ such that $\Omega \cap B^{R}=W_{S}^{R}\left(\Pi_{k}\right)$. Let $u$ be a positive solution of $(1.1)$ in $\Omega$ such that $u \rightarrow \infty$ locally uniformly at $\partial \Omega \cap B^{R}$. Then, for every $R^{\prime}, 0<R^{\prime}<R$,

$$
u(x) /(\sqrt{k(x)} r)^{-2 /(p-1)} w(\sigma) \rightarrow 1 \quad \text { as } \operatorname{dist}\left(x, \partial \Omega \cap B^{R^{\prime}}\right) \rightarrow 0
$$

where $w$ is the large solution of Equation (2.3) and $x$ is given by $(r, \sigma, z)$ in cylindrical coordinates with axis $\Pi_{k}$.

Proof. - This is a consequence of Proposition 2.4 and Theorem 3.1.

Lemma 3.4. - Let $\Omega$ be a bounded domain with piecewise $C^{1}$ boundary and let $u$ be the large solution of (1.1) in $\Omega$. Suppose that $\Gamma$ is a connected 
curved edge of dimension $k$ on $\partial \Omega$. For $P \in \Gamma$, let $W_{S^{\prime}}\left(\Pi_{k}\right)$ be the limiting wedge at $P$ (in an appropriate local set of coordinates) and let $w_{p}$ be the large solution of Equation (2.3) with $S=S^{p}$. Then,

$$
u(x) /(\sqrt{k(P)} r)^{-2 /(p-1)} w_{P}(\sigma) \rightarrow 1 \quad \text { as } x \rightarrow P, x \in \Omega .
$$

Iocally uniformly with respect to $\sigma$ in $S_{P}$ and with respect to $P$ on $\Gamma$. Here $(r, \sigma, z)$ is a set of cylindrical coordinates with axis $\Pi_{h}$ corresponding to the local set of coordinates mentioned above and $x=(r, \sigma, z)$.

Proof. - Let $\left\{S_{n, 1}^{P}\right\}$ be a monotone increasing sequence of domains on $S^{N-k-1}$ such that $\bar{S}_{n, 1}^{P^{P}} \subset S^{P}$ and $S_{n, 1}^{P} \rightarrow S^{P}$. Let $\left\{S_{n, 2}^{P}\right\}$ be a monotone decreasing sequence of domains on $S^{N-k-}$ such that $S_{n, 2}^{P^{\prime}} \supset \bar{S}^{P}$ and $S_{n, 2}^{P} \rightarrow S^{P}$. In addition assume (as we may) that $\left\{S_{n, i}^{P}\right\} i=1.2$, is uniformly of class $C_{y t}^{0,1}$. If $\left\{R_{n}\right\}$ is a sequence of numbers monotonically decreasing to zero denote,

$$
D_{n, i}^{P^{r}}=W_{s_{n, i}}^{R_{2}}\left(\Pi_{k}\right) . \quad i=1,2, \quad n=1,2, \ldots
$$

From the definition of limiting wedge it follows that, given $\left\{R_{n}\right\}$, one can choose the sequences $\left\{S_{n, i}^{P}\right\}$ in such a way that the domains $D_{n, i}^{P}$, defined by (3.6) in an appropriate set of local coordinates centered at $P$. satisfy the following relations,

$$
D_{n, 1}^{l^{\prime}} \subset \Omega \cap B^{R_{\prime \prime}}(P) \subset D_{n, 2}^{P,}, \quad n=1,2 \ldots
$$

Let $v_{n .1}^{P}$ be the large solution of (1.1) in $D_{n, 1}^{P}$. Let $v_{n, 2}^{P}$ be a positive solution of (1.1) in $D_{n, 2}^{P}$ such that $v_{n, 2}^{P}(r, \sigma, z) \rightarrow 0$ as $R \rightarrow R_{n}$, uniformly with respect to $\sigma$ in $S_{n, 2}^{P}$ and with respect to $z,|z|<R_{n}$ and $v_{n, 2}^{P} \rightarrow \infty$ locally uniformly on the remaining portion of $\partial D_{n, 2}^{P}$. Such a solution can be obtained as the limit of a monotone increasing sequence of bounded solutions of (1.1) in $D_{n, 2}^{P}$ and we shall assume that $v_{n, 2}^{P}$ is of this type. Then

$$
v_{n, 2}^{P} \leq u \leq v_{n, 1}^{P} . \quad n=1,2, \ldots
$$

Further, if $w_{n, i}^{P}$ is the large solution of Equation (3.3) in $S_{n, i}^{P}$, then by Lemma 3.3,

$v_{n, i}^{P}(x) /(\sqrt{k(x)} r)^{-2 /(p-1)} w_{n, i}^{P}(\sigma) \rightarrow 1 \quad$ as $\operatorname{dist}\left(x, \partial D_{n, i}^{P}\right) \rightarrow 0$

for $|x| \leq R_{n} / 2, i=1,2$ and every $n$. Finally, by Proposition 2.7,

$$
\lim _{n \rightarrow \infty} w_{n, i}^{P}=w_{P} \quad \text { locally uniformly in } S^{P}, \quad i=1,2 .
$$


From (3.8)-(3.10) it follows that for every $\varepsilon>0$ and every compact subset $A$ of $S^{P}$, there exists a positive number $r_{\varepsilon}=r_{\varepsilon}(A, P)$ such that, for $\sigma \in A$,

$$
\left.\begin{array}{c}
1-\varepsilon \leq u(x) /(\sqrt{k(P)} r)^{-2 /(p-1)} w_{P}(\sigma) \leq 1+\varepsilon \\
\text { for }|x-P| \leq r_{\varepsilon}(A, P)
\end{array}\right\}
$$

Since $\Omega$ is piecewise $C^{1}, S^{P}$ varies smoothly with $P$ in $\Gamma$. In particular, if $P \rightarrow P_{0} \in \Gamma$ then $S^{P} \rightarrow S^{P_{0}}$ and there is a neighborhood $M$ of $P_{0}$ such that $\left\{S^{P}: P \in M \cap \Gamma\right\}$ is uniformly of class $C_{g r}^{0,1}$. Therefore, if $\left\{R_{n}\right\}$ is a sequence of positive numbers monotonically decreasing to zero, one can choose the sequences $\left\{S_{n, i}^{P}\right\}_{n=1}^{\infty}, i=1,2$, for each $P$ in $M \cap \Gamma$, in such a way that,

(i) $S_{n, i}^{P} \rightarrow S^{P}$ uniformly with respect to $P \in M \cap \Gamma, i=1,2$;

(ii) for each $P \in M \cap \Gamma$ there is a set of local coordinates centered at $P$ and varying smoothly with $P$, such that the sets $D_{n, i}^{\Gamma}$ given by (3.6) in this set of coordinates, satisfy (3.7);

(iii) the family $\left\{S_{n, \imath}^{\Gamma}: P \in M \cap \Gamma, i=1,2, n=1,2, \ldots\right\}$ is uniformly of class $C_{g r}^{0,1}$.

Consequently, the relations (3.8)-(3.10) hold for each $P \in M \cap \Gamma$; in addition the convergence in (3.10) is uniform with respect to $P$ as above, while the convergence in (3.9) is uniform in $P$, for each fixed $n$.

The statement concerning (3.9) can be verified as follows. In view of (iii) and Proposition 1.8 the functions $w_{n, i}^{P}$ blow up uniformly with respect to $n, i$ and $P \in M \cap \Gamma$. Therefore, following the arguments in the proof of Theorem 2.2 and Proposition 2.4, we find that the convergence statement of Lemma 3.3, applied to the domains $D_{n, i}^{P}$ (with fixed $n$ ) holds uniformly with respect to $P \in M \cap \Gamma$. Thus the convergence in (3.9) is uniform in $P$, for each fixed $n$.

The uniform convergence in (3.10) should be understood as follows. Suppose that $A$ is a compact set on $S^{N-k-1}$ which is contained in $S^{P}$ for every $P \in M \cap \Gamma$. Then $\lim _{n \rightarrow \infty} w_{n, i}^{P}=w_{P}$ uniformly with respect to $\sigma$ in $A$ and with respect to $P \in M \cap \mathrm{l}$. To verify (3.10), assume that the convergence is not uniform with respect to $P$ (say, for $i=1$ ). Then there exists a sequence of points $P_{k} \in M \cap \Gamma$, a sequence $n_{k} \rightarrow \infty$ and a positive $\varepsilon$ such that,

$$
\begin{array}{cl}
\sup _{A}\left|\tilde{w}_{k}-w_{P_{k}}\right| \geq \varepsilon \quad \text { where } \quad \tilde{w}_{k}=w_{n, 1}^{P} \\
\text { with } n=n_{k} \quad \text { and } \quad P=P_{k}, k=1,2, \ldots
\end{array}
$$


We may assume that $\left\{P_{k}\right\}$ converges to some point $P_{0}$ in $M \cap \Gamma$. Hence $S^{P_{k}} \rightarrow S^{P_{0}}$ and consequently $w_{P_{k}} \rightarrow w_{P_{0}}$ uniformly in $A$. In addition, from (i) we deduce that $S_{n_{k}, 1}^{P_{k}} \rightarrow S^{P_{0}}$ so that $\tilde{w}_{k} \rightarrow w_{P_{n}}$ uniformly in $A$. Thus we reach a contradiction.

In view of these observations we conclude that the number $r_{\varepsilon}(A, P)$ in (3.11) can be chosen independently of $P$ in some neighborhood of $P_{0}$, provided that $A$ is a compact subset of each domain $S^{P}$ with $P$ in this neighborhood. This concludes the proof of the lemma.

Next we bring a technical lemma that will be used in the derivation of the main result of this section.

Lemma 3.5. - Let $\Omega$ be a piecewise $C^{1}$ domain. Suppose that $0 \in \partial \Omega$ and that there exists a ball $B^{R}$ centered at the origin such that $\Omega \cap B^{R}=$ $W_{S}^{R}\left(\Pi_{k}\right)$. Suppose that $\sigma_{0}$ is a point on $\partial S$ such that, for some positive $r_{0}$, the set $\left\{\left(r, \sigma_{0}, 0\right): 0<r<r_{0}\right\}$ lies on a 'linear' edge of dimension $k_{0}$. (Necessarily, $k_{0} \leq N-k-2$.) Thus there exists a spherical cap $B_{0}$ on $S^{N-k-1}$, centered at $\sigma_{0}$, and a domain $\sum$ on $S^{N-k-k_{0}-1}$ such that

$$
\begin{aligned}
& \left\{(r, \sigma, 0): 0<r<r_{0}, \sigma \in B_{0}\right\} \cap \Omega \\
& \quad=\left\{(r, \sigma, 0): 0<r<r_{0}, \sigma \in B_{0}\right\} \cap W_{\Sigma}\left(\Pi_{k_{0}}\right)
\end{aligned}
$$

where $W_{\Sigma}\left(\Pi_{k_{0}}\right)$ is a wedge in $\mathbb{R}^{N-k}$ (identified here with the subspace $z=0$ of $\mathbb{R}^{N}$ ) whose axis contains the ray $\left\{\left(r, \sigma_{0}, 0\right): 0<r\right\}$. Let us denote this set by $\tilde{W}$ and its cross-section (for fixed $r$ ) by $\tilde{W}_{r}$. In an appropriate local set of 'cylindrical' coordinates in $S^{N-k-1}$, with axis $\Pi^{\prime}=\left\{\sigma:(r, \sigma, 0) \in \Pi_{k_{0}}\right.$ for $r>0\}$, say $(\rho, \theta, \zeta)$ with $\zeta \in \Pi^{\prime}$, we have,

$$
\tilde{W}_{r}=\left\{(r, \sigma, 0): \sigma=(\rho, \theta, \zeta), 0<\rho<\gamma(\zeta) r, \theta \in \Sigma, \zeta \in \Pi^{\prime}\right\}
$$

where $\gamma$ is a smooth, non-negative function of $\zeta$ which is positive at $\zeta=0$. (It is determined by $B_{0}$ and $\Pi^{\prime}$.)

Let $w$ be the large solution of Equation (2.3) in $S$ and let $w^{*}$ be the large solution of (2.3) in $\Sigma$, with $\lambda(N-k-1, p)$ replaced by $\lambda\left(N-k-k_{0}-1, p\right)$. Then if $Q$ is a point on $\partial \Omega$ lying on the edge of $\tilde{W}$ and $u$ is a large solution in $\Omega$ we have,

$$
\begin{aligned}
& \left.\begin{array}{c}
u(x) /(\sqrt{k(0)} r)^{-2 /(p-1)} w(\sigma) \rightarrow 1 \\
\text { as } x \rightarrow 0, \quad x=(r, \sigma, z)
\end{array}\right\} \\
& \left.\begin{array}{c}
u(x) /(\sqrt{k(Q)} r \rho)^{-2 /(p-1)} w^{*}(\theta) \rightarrow 1 \\
\text { as } x \rightarrow Q, \quad x=(r, \rho, \theta, \zeta, z) .
\end{array}\right\}
\end{aligned}
$$


The rate of convergence does not depend on $Q$ but only on $|x-Q| / r$ i.e. on $\rho$. Hence, letting $Q$ tend to the origin we obtain,

$$
w(\sigma) / \rho^{-2 /(p-1)} w^{*}(\theta) \rightarrow 1 \text { as } \rho \rightarrow 0 \text {. }
$$

Proof. - This is a consequence of Lemma 3.3 which, in the present case, will be applied at the origin (to obtain $(3.12)_{1}$ ) and at $Q$ (to obtain $\left.(3.13)_{2}\right)$.

Definition 3.6. - A wedge $W_{S}\left(\Pi_{k}\right)$ is of order one if $S$ is of class $C^{1}$. Similarly, a $k$-dimensional curved edge is said to be of order one if (in the notation of Definition 3.2 (i)) $S$ is $C^{1}$. A piecewise $C^{1}$ domain $\Omega$ in $\mathbb{R}^{N}$ (or in $S^{N-1}$ ) is said to be of order one if it is not $C^{1}$ and if every $k$-dimensional curved edge on $\partial \Omega$ (for every $k$ ) is of order one. Inductively we define: a wedge $W_{S}\left(\Pi_{k}\right)$ is of order $m$ if $S$ is of order $m-1$. A piecewise $C^{1}$ domain $\Omega$ in $\mathbb{R}^{N}$ (or in $S^{N-1}$ ) is said to be of order $m$ if every $k$-dimensional curved edge on $\partial \Omega$ (for every $k$ ) is of order $m^{\prime} \leq m$ and there exists on $\partial \Omega$ a curved edge of order $m$.

Theorem 3.7. - Let $\Omega$ be a bounded piecewise $C^{1}$ domain. Then there exists a unique large solution $u$ of (1.1) in $\Omega$.

If $P \subset \partial \Omega$, let $W_{S^{P}}\left(\Pi_{k}\right)$ be the limiting wedge at $P$ and let $w_{P}$ denote the unique large solution of Equation (3.3) with $S=S^{P}$. (We shall use this notation for every $P$ on $\partial \Omega$, even if the boundary is $C^{1}$ in a neighborhood of $P$. As we have mentioned before, in such a case $k=0$ and $S^{P}$ is a half sphere.) Let $(r, \sigma, z)$ denote a local set of cylindrical coordinates with axis $\Pi_{k}$ such that $W_{S^{+}}\left(\Pi_{k}\right)=\left\{(r, \sigma, z): 0<r, \sigma \in S^{P}, z \in \Pi_{k}\right\}$. With this notation the solution $u$ satisfies,

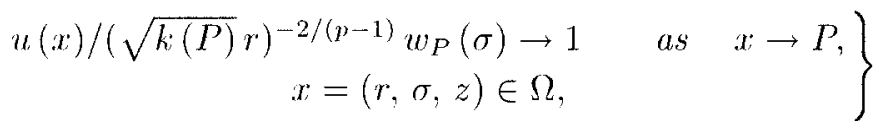

and the convergence is uniform with respect to $P \in \partial \Omega$.

Proof. - It is sufficient to consider the case where $h=0$ and $k-1$, i.e. $\Delta u=u^{P}$. Indeed, if (3.14) is valid in this special case, then (by Proposition 2.4) it is also valid in the general case of Equation (1.1). The proof will be by induction on the order of $\Omega$.

If $P$ is a point on $\partial \Omega$ such that the boundary is $C^{2}$ in a neighborhood of $P$ statement (3.14) is well known although in a slightly different form (see $[\mathrm{BM} 1,2]$ or $[\mathrm{V} 1])$, namely,

$$
u(x) / c_{p} \delta(x)^{-2 /(p-1)} \rightarrow 1 \quad \text { as } \delta(x)=\operatorname{dist}(x, \partial \Omega) \rightarrow 0
$$


In this case the denominator in (3.14) (with $k: \equiv 1$ ) is the large solution for a half space, specifically the half space bounded by the tangent plane to $\partial \Omega$ at $P$. which contains the intersection of $\Omega$ with a neighborhood of $P$. This solution is also given by $U(x)=c_{p} \delta(x)^{-2 /(p-1)}$, where $\delta(x)$ is the distance from $x$ to the langent plane. Therefore (3.14) and (3.15) are equivalent.

A modification of the arguments employed in the proof of (3.15) (as presented in $[\mathrm{M}]$ ) shows that the result holds under the assumption that $\partial \Omega$ is of class $C^{1}$. Indeed (3.15) is obtained by comparing $u$ with the large solution in a ball touching $\partial \Omega$ at $P$ (for an upper estimate) and in an annulus whose inner boundary touches $\partial \Omega$ at $P$ (for a lower estimate). For this reason it is required that $\Omega$ satisfies the interior and the exterior ball conditions. However if one replaces the ball and the annulus by conical sections with vertex at $P$, the same argument leads to $(3.15)$ and requires only $C^{1}$ boundary.

Next suppose that $\Gamma$ is a $k$-dimensional curved edge of order 1 on $\partial \Omega$ and let $P_{0} \in \mathrm{T}$. Then, in the notation of Definition 3.2,S and $S^{P_{u}}$ are of class $C^{1}$ and $\partial \Omega \backslash \Gamma$ is $C^{\perp}$ in a neighborhood $M$ of $P_{0}$. Using further the notation of Definition 3.2, let $D_{i s}$ denote a one-sided neighborhood of $\partial \Omega \cap M$ which is the pre-image by $T$ of the set $T(M) \cap W_{S_{3}}\left(\Pi_{k}\right)$ where $S_{3}=\{\sigma \in S:$ dist $(\sigma, \partial S)<\beta\}$. It follows from (3.15) that, if $\beta$ is sufficiently small, (3.14) holds for $P \in \Gamma \cap M$ and $x \in D_{i,}$, uniformly with respect to such $P$. ( $\beta$ must only be small enough so that for $x \in D_{3,}, \delta(x)<|x-P|$.) On the other hand, by Lemma 3.4, (3.14) holds in $(M \cap \Omega) ! D_{3}$ uniformly with respect to $P \in \Gamma \cap M$. Consequently (3.14) holds uniformly with respect to $P \in \Gamma \cap M$. Thus the theorem holds (for equation $\Delta u=u^{p}$ ) in domains of order 1 in $\mathbb{R}^{N}$. Hence (by Proposition 2.4) it holds for any equation of the form (1.1), in domains of order 1 in $\mathbb{R}^{N}$ or in $S^{N-1}$.

Now suppose that the theorem holds for domains of order $m^{\prime}<m$ and let $\Omega$ be a domain in $\mathbb{R}^{N}$ of order $m$. Observe that this assumption implies that if $\Omega$ is an arbitrary, piecewise $C^{1}$ domain and if $P$ is a point on $\partial \Omega$ lying on a curved edge of order $m !<m$ then (3.14) holds in a neighborhood of $P$. (This is again a consequence of Proposition 2.4.) As before we shall consider the equation $\Delta u=u^{p}$. Suppose that $\Gamma$ is a $k$-dimensional curved edge of order $m$ on $\partial \Omega$ and let $P_{0} \in I$. Then, in the notation of Definition 3.2, $S$ is a domain of order $m-1$ and there is a neighborhood $M$ of $P_{0}$ such that if $Q \in(\partial \Omega \backslash \Gamma) \cap M$, either $\partial \Omega$ is $C^{1}$ in a neighborhood of $Q$, or $Q$ lies on a curved edge of order $m^{\prime}<m$. In either case, our assumption implies that (3.14) holds at each such point $Q$. This 
fact and Lemma 3.5 imply that (for $D_{\beta}$ as in the previous paragraph), if $\beta$ is sufficiently small, (3.14) holds for $P \in \Gamma \cap M$ and $x \in D_{\beta}$, uniformly with respect to such $P$. (In fact (3.14) with respect to $Q$ corresponds to $(3.12)_{2}$ with $\Sigma=\Sigma_{Q}$. We note also that if $Q \rightarrow P$ along a curved edge then $\Sigma_{Q}$ converges to a donain $\Sigma$ such that $W_{\Sigma}\left(\Pi_{k_{0}}\right)$ is a limiting edge al a point $\sigma_{0}$ on $\partial S^{P}$.) The proof may now be completed using Lemma 3.4, as in the previous paragraph.

\section{ESTIMATES AT A RE-ENTRANT CUSP}

In this section we present estimates for the growth of large solutions of (1.1) in the neighborhood of a boundary point of $\Omega$ which is the vertex of a re-entrant cusp. We shall assume that $\Omega$ is bounded, of class $C_{g r}$ and that $\partial \Omega$ satisfies the exterior cone condition away from the cusp. Without loss of generality we shall assume that the vertex of the cusp is at the origin and that there exist $R>0$ such that $\Omega_{R}=\Omega \cap B_{R}(0)$ is a simply connected domain satisfying the following conditions:

$$
\begin{aligned}
\Omega_{R} & =\left\{(r, \sigma): 0<r<R, \sigma \in \Gamma_{r}\right\}, \quad \Gamma_{r} \subset S_{N-1}, \\
\operatorname{diam} \bar{\Gamma}_{r}^{c} & \rightarrow 0 \quad \text { as } r \rightarrow 0 \quad \text { and } \bigcap_{0<r<R} \bar{\Gamma}_{r}^{c} \text { is not empty. }
\end{aligned}
$$

Let $P=\bigcap_{0<r<R} \bar{\Gamma}_{r}^{c}$ and set

$$
\theta_{0}(r)=\sup \left\{|\sigma|_{P}: \sigma \in \bar{\Gamma}_{r}^{c}\right\}, \quad \theta(r)=\sup _{0<s \leq r} \theta_{0}(s) .
$$

where $|\sigma|_{P}=d_{g}(\sigma, P)$ is the geodesical distance between $\sigma$ and $P$ on $S^{N-1}$. Finally denote

and

$$
\left.\begin{array}{l}
\Xi_{p}=\left\{\sigma \in S^{N} 1:|\sigma|_{P}>\rho\right\} \\
G_{r}=\left\{(s, \sigma): 0<s<r, \sigma \in \Xi_{\theta(r)}\right\} .
\end{array}\right\}
$$

Thus

$$
G_{r} \subset \Omega_{r} . \text { for } 0<r \leq R \text {. }
$$

Proposition 4.1. - (i) Suppose that $1<p<(N-1) /(N-3)$. Then there exists a large solution of (1.1) in $\Omega$. If $u$ is such a solution. its growth near the cusp is given by,

$$
\lim _{r \rightarrow 0}(\sqrt{k(0)} r)^{2 /(p-1)} u(r, \sigma)=w_{0}(\sigma)
$$


where (in the notation of (1.12)) $w_{0}=w_{S}$ with $S=S^{N-1} \backslash\{P\}$.

(ii) Suppose that $N>3$ and that $p \geq(N-1) /(N-3)$. If $u$ is a positive solution of (1.1) in $\Omega$ then,

$$
\lim _{r \rightarrow 0} r^{2 /(p-1)} u(r, \sigma)=0 .
$$

Proof. - (i) Our assumptions on $\Omega$ imply that it satisfies the exterior segment condition. Therefore the existence of a large solution follows from Remark 1.9. In view of Proposition 2.4, it is sufficient to establish (4.5) in the special case where (1.1) reduces to the equation $\Delta u=u^{p}$ and $\Omega=\Omega_{R}$. If $R$ is sufficiently small then $\Omega_{R} \subset D_{P}=\left\{(r, \sigma): 0<r, \sigma \in S^{N-1}\right.$, $\sigma \neq P\}$. The function $u_{S}$ given by (1.12) is a large solution of $\Delta u=u^{p}$ in $D_{P}$. Hence $u_{S} \leq u$ and

$$
\liminf _{r \rightarrow 0} r^{2 /(p-1)} u(r, \sigma) \geq w_{0}(\sigma)
$$

On the other hand if $v_{R}$ is a large solution of $\Delta v=v^{p}$ in $G_{R}$ then (in view of (4.4)), $v_{R} \geq u$. (Note that our assumptions imply that the large solution in $\Omega$ is unique so that it is sufficient to verify that $v_{R}$ dominates every bounded positive solution in $\Omega$.) By Proposition $2.4, v_{R}$ behaves near the origin in the same way as the solution of $\Delta u=u^{p}$ in $\{(s, \sigma): 0<s$, $\left.\sigma \in \Xi_{\theta(R)}\right\}$. Therefore by (1.12)

$$
\limsup _{r \rightarrow 0} r^{2 /(p-1)} u(r, \sigma) \leq w_{R}(\sigma)
$$

where $w_{R}$ is the large solution of (1.13) in $\Xi_{\theta(R)}$. Note that $\Xi_{\theta(R)} \uparrow$ $S^{N-1} \backslash\{P\}$ as $R \downarrow 0$ so that $w_{R} \downarrow w_{0}$. (Here we use the fact that $w_{0}$ is the unique large solution of (1.13) in $S^{N-1} \backslash\{P\}$, see [V2, 3].) Hence (4.7) and (4.8) imply the stated result.

(ii) Inequality (4.8) holds by the same argument as in the first part. However when $p \geq(N-1) /(N-3)$ the singularity of $w_{0}$ at $P$ is removable [BV]. The only non-negative solution of (1.13) on the whole sphere $S^{N-1}$ is $w \equiv 0$. (Note that in this case $\lambda(N, p) \leq 0$.) Thus (4.8) implies (4.6).

In the remaining part of the section we derive more precise estimates for the behavior of large solutions in the neighborhood of the cusp, in the case where $p \geq(N-1) /(N-3)$. We start with estimates from above.

THEOREM 4.2. - Suppose that the function $\theta(\cdot)$ defined in (4.2) is continuous.

(i) Let $p>(N-1) /(N-3)$. Given $r_{0} \in(0, R)$ and $\tau>1$, there exists a constant $c\left(r_{0}, \tau\right)$ (depending also on $N, p, k, h$ ) such that every positive 
solution $u$ of (1.1) in $\Omega$ satisfies the inequality,

$$
\begin{gathered}
u(r, \sigma) \leq c\left(r_{0}, \tau\right)|\sigma|_{P}^{3}{ }^{N} \nu(r)^{-2 /(p-1)}, \\
\text { for } 0<r<r_{0}, \sigma \in \Lambda_{\tau, r},
\end{gathered}
$$

where $\nu$ is a strictly monotone increasing function in $\mathbb{R}_{+}$whose inverse is given by,

$$
\nu^{-1}(\rho)=\rho \theta(\rho)^{-1+(p-1)(N-3) / 2}
$$

and

$$
\Lambda_{\tau, r}=\left\{\sigma \in S^{N-1}:|\sigma|_{P}>\tau \theta(\nu(r))\right\} .
$$

Note that as $r \downarrow 0, \rho=\nu(r) \downarrow 0$ and consequently $\Lambda_{\tau, r} \uparrow S^{N-1} \backslash\{P\}$.

(ii) Let $p=(N-1) /(N-3)$. Given $r_{0} \in(0, R)$ and $\bar{s} \in(0, \pi)$, there exists a constant $c^{\prime}\left(r_{0}, \bar{s}\right)$ (depending also on $N, p, k, h$ ) such that every positive solution $u$ of (1.1) in $\Omega$ satisfies the inequality,

$$
u(r, \sigma) \leq c^{\prime}\left(r_{0}, \bar{s}\right) \nu(r)^{3-N}, \quad \text { for } 0<r<r_{0}, \sigma \in \Xi_{\bar{s}},
$$

where

$$
\nu^{-1}(\rho)=\rho\left(\log (1 / \theta(\rho))^{-1 / 2} .\right.
$$

Remark 4.3. - Note that the expression $-1+(p-1)(N-3) / 2$ is positive in case (i) and vanishes when $p=(N-1) /(N-3)$. Since by its definition $\theta($.$) is monotone increasing, the right hand side of (4.10)$ or $(4.10)^{\prime}$ is strictly monotone. Thus $\nu$ is well defined. Further note that,

$$
r / \nu(r) \rightarrow 0 \text { as } r \rightarrow 0
$$

Indeed if $\nu(r)=\rho$ then,

$$
r / \rho= \begin{cases}\theta(\rho)^{-1+(p-1)(N-3) / 2} & \text { in case (i) } \\ \left(\log (1 / \theta(\rho))^{-1 / 2}\right. & \text { in case (ii) }\end{cases}
$$

By $(4.1)_{2} \lim _{\rho \rightarrow 0} \theta(\rho)=0$ and by (4.10) or $(4.10)^{\prime} \rho \rightarrow 0$ as $r \rightarrow 0$. Therefore (4.13) implies (4.12). In view of (4.9) and (4.9)' this fact implies that the rate of blow up of large solutions at a cusp is lower than the rate of blow up at regular points or corners.

The proof of the theorem is based on the following lemma.

Lemma 4.4. - Consider the equation,

$$
\Delta_{g} w-\lambda w-\kappa w^{p}=0,
$$

where $\lambda, \kappa$ are constants, $\kappa>0$ and $\Delta_{g}$ is the Laplace-Beltrami operator on $S^{N-1}$. Let $w_{\eta}$ denote the large solution of (4.14) in $\Xi_{\eta}$. 
(i) If $p>\frac{N-1}{N-3}$ then, for every $\tau>1$ there exists a constant $c_{1}(\tau)$ (which depends also on $N, p, r, \lambda)$ such that,

$$
w_{\eta}(\sigma) \leq c_{1}(\tau)|\sigma|_{P}^{3-N} \eta^{N-3-\frac{2}{\eta-1}}, \quad \forall \sigma \in \Xi_{\tau \eta}: \quad 0<\eta<\pi / \tau .
$$

(ii) If $p=\frac{N-1}{N-3}$ and $\lambda \geq 0$ then, for every $\bar{s} \in(0, \pi)$ there exists a constant $c_{2}(\bar{s})$ (which depends also on $\left.N, p, \kappa, \lambda\right)$ such that,

$$
w_{\eta}(\sigma) \leq c_{2}(\bar{s})\left(\log \frac{1}{\eta}\right)^{(3-N) / 2}, \quad \forall \sigma \in \Xi_{\bar{s}}, \quad 0<\eta<\bar{s} / 2
$$

The proof of the lemma is given in the appendix. We turn now to,

Proof of Theorem 4.2. - For $\rho \in(0, R)$ let $\psi_{\rho}$ be the unique large solution of

$$
\Delta \psi-\kappa \psi^{p}=0 \text { in } B_{p}(0)
$$

and let $w_{\rho}$ be the unique large solution of

$$
\Delta_{g} w-\lambda w-\kappa w^{p}=0, \quad \text { in } \Xi_{\theta(\rho)},
$$

where $-\lambda=\lambda(N, p)$ as in (1.13) and $\kappa=k(0) / 2$. We observe that in the case $p=(N-1) /(N-3), \lambda>0$. Then the function $u_{\rho}$ given by

$$
u_{p}(r, \sigma)=r^{-\frac{2}{p-1}} w_{\rho}(\sigma) \text {, }
$$

is a large solution of (4.17) in the cone $\left\{(r, \sigma): 0<r, \sigma \in \Xi_{\theta(p)}\right\}$. Consequently $U_{\rho}=u_{\rho}+\psi_{p}$ is a supersolution of (4.17) in $G_{p}$,

$$
\Delta U_{p}-\kappa U_{p}^{p}<0 \text {. }
$$

Note that (by scaling) $\psi_{p}(r) \geq \psi_{1}(0) \rho^{-2 /(p-1)}$. Therefore there exists $\rho_{0}>0$ such that for $\rho \in\left(0, \rho_{0}\right), U_{p}$ is sufficiently large so that (in view of $(4.20)$ ),

$$
\Delta U_{\rho}+h U_{p}-k U_{p}^{p}<0 \text { in } G_{\rho}
$$

Since $U_{\rho}$ blows up on $\partial G_{\rho}$ we conclude that,

$$
u(r, \sigma) \leq U_{\rho}(r, \sigma)=r^{-\frac{2}{p-1}} w_{\rho}(\sigma)+\psi_{\rho}(r) \text { in } G_{\rho}
$$

From (4.22), Lemma 4.4 and the standard estimate for large solutions in regular domains it follows that in case (i),

$$
u(r, \sigma) \leq c_{1}(\tau)|\sigma|_{P}^{3-N} \theta(\rho)^{N-3-\frac{2}{p-1}} r^{-\frac{2}{p-1}}+c_{2}(\rho-r)^{-\frac{2}{p-1}}
$$


for $0<r<\rho \leq \rho_{0}$ and $\sigma \in \Xi_{\tau \theta(\rho)}$, and in case (ii),

$$
u(r, \sigma) \leq c_{2}(\bar{s})\left(\log \frac{1}{\theta(\rho)}\right)^{(3-N) / 2} r^{3-N}+c_{2}(\rho-r)^{3-N},
$$

for $0<r<\rho \leq \rho_{0}$ and $\sigma \in \Xi_{\bar{s}}$. The constants $c_{1}, c_{2}$ are independent of $\rho$ which can be freely chosen in the interval $\left(0, \rho_{0}\right)$. We shall choose $\rho$ in such a way that the two additive terms on the right hand side of (4.23) (resp. $\left.(4.23)^{\prime}\right)$ will be of the same order of magnitude when $r \rightarrow 0$. Thus, assuming that $0<r<\rho / 2$, we choose $\rho$ so that,

$$
\begin{gathered}
\theta(\rho)^{N-3-\frac{2}{p-1}} r^{-\frac{2}{p-1}}=\rho^{-\frac{2}{p-1}}, \quad \text { in case (i), } \\
\left(\log \frac{1}{\theta(\rho)}\right)^{(3-N) / 2} r^{3-N}=\rho^{3-N}, \text { in case (ii). }
\end{gathered}
$$

With this choice of $\rho$ we obtain (4.9) and (4.9)'. (Note that, with $\rho=\nu(r)$, $\left.\Xi_{\tau \theta(\rho)}=\Lambda_{\tau, r}.\right)$

Examples 4.5. - (a) Suppose that $\theta(\rho)=\rho^{1+\delta}$ for some $\delta>0$. If $p>\frac{N-1}{N-3}$ then (4.9) yields,

$u(r, \sigma) \leq c\left(r_{0}, \tau\right)|\sigma|_{P}^{3-N} r^{-\frac{2}{(p-1)(1+\delta, H)}}, \quad \forall \sigma \in \Lambda_{\tau, r}, 0<r<r_{0}$,

where $\mu=((p-1)(N-3) / 2)-1$. Note that in this case $\mu>0$.

If $p=\frac{N-1}{N-3}$ (and $N>3$ ) then (4.9)' yields,

$$
\left.\begin{array}{c}
u(r, \sigma) \leq c^{\prime}\left(r_{0}, \bar{s}\right) r^{3-N}\left(\log \frac{1}{r}\right)^{(3-N) / 2}, \\
\vee \sigma \in \Xi_{\bar{s}}, \quad 0<r<r_{0}
\end{array}\right\}
$$

(b) Suppose that $\theta(\rho)=e^{-\beta / \rho}$ for some $\beta>0$. If $p>\frac{N-1}{N}$ then (4.9) yields,

$$
\left.\begin{array}{c}
u(r, \sigma) \leq c\left(r_{0}, \tau\right)|\sigma|_{P}^{3-N} \beta\left(\log \frac{1}{r}\right)^{\frac{2}{p-1}}, \\
\forall \sigma \in \Lambda_{\tau, r}, \quad 0<r<r_{0} .
\end{array}\right\}
$$

If $p=\frac{N-1}{N-3}$ then $(4.9)^{\prime}$ yields,

$$
\left.\begin{array}{c}
u(r, \sigma) \leq c^{\prime}\left(r_{0}, \bar{s}\right) \beta^{(3-N) / 3} r^{\frac{2}{3}(3-N)} \\
\forall \sigma \in \Xi_{\bar{s}}, \quad 0<r<r_{0}
\end{array}\right\}
$$

Next we derive a lower estimate for the behavior of large solutions near a cusp. Here we shall consider a more restricted family of cusps. Specifically 
we shall assume that for some $R>0, \Omega_{R} \subset D_{R}=D \cap B_{R}(0)$, where (in some local set of coordiates $z$ obtained from $x$ by rotation)

$$
D=\left\{\left(z^{\prime}, z_{N}\right) \in \mathbb{R}^{N-1} \times \mathbb{R}_{+}:\left|z^{\prime}\right| \geq \phi\left(z_{N}\right)\right\} \cup\left\{z: z_{N}<0\right\},
$$

and $\phi$ is a convex function in $C^{1}([0, \infty))$ such that $\phi(0)=0, \phi^{\prime}(0)=0$ and $\phi(\rho)>0$ for $\rho>0$. For $\rho>0$, let $P_{\rho}$ denote the intersection of the tangent to the curve $\phi$ at the point $\rho$ with the $z_{N}$ axis. Thus $P_{\rho}=\left(0, \zeta_{\rho}\right)$ where $\zeta_{\rho}=\rho-\left(\phi(\rho) / \phi^{\prime}(\rho)\right)$. We denote by $P$ the point $(0,1)$ (i.e. $z^{\prime}=0$, $z_{N}=1$ ) and observe that $P \in \bar{\Gamma}_{r}^{c}, 0<r<R$ (in the notation introduced at the beginning of the section.)

THEOREM 4.6. - Let $\Omega$ be a domain as before and assume that the cusp satisfies the conditions described above. In addition suppose that $p \geq(N+1) /(N-3)$ and that $h \geq 0$. Then there exists a positive constant $c^{\prime}$, depending on $N$, p such that every maximal solution $u$ of (1.1) in $\Omega$ satisfies the inequality,

$$
u(z) \geq c^{\prime}\left|z-P_{\rho}\right|^{-\frac{2}{p-1}} \phi^{\prime}(\rho)^{N-3-\frac{2}{p-1}},
$$

for every $\rho$ in $(0, R)$ and every $z$ in $\Omega_{R}$ such that $\frac{z-P_{\rho}}{\left|z-P_{\rho}\right|} \in \Xi_{\phi^{\prime}(\rho)}$.

For the definition of maximal solutions see Remark 1.4 (ii). Under the assumptions of the present theorem, it is not known if there exists a large solution in the sense of (1.2). However it is clear that a maximal solution exists. The proof of the theorem is based on the following lemma, whose proof will be given in the appendix.

Lemma 4.7. - Suppose that $p>\frac{N-1}{N-3}$. For $\eta>0$, let $w_{\eta}$ be the unique large solution of

$$
\Delta_{g} w-\lambda w-\bar{k} w^{p}=0, \quad \text { in } \Xi_{\eta},
$$

where $\lambda$ and $\bar{k}$ are constants, $\bar{k}>0$ and $\lambda \leq(N-1)(N-3) / 4$. Then there exists a positive constant c (depending on $N, p$ ) such that,

$$
w_{\eta}(\sigma) \geq c \eta^{N-3-\frac{2}{p-1}}, \quad \text { for every } \sigma \in \Xi_{\eta}, \quad 0<\eta<\pi
$$

Proof of Theorem 4.6. - Let $\rho \in(0, R)$ and let $\left(r_{\rho}, \sigma\right)$ denote spherical coordinates centered at $P_{\rho}$. Let $\alpha(\rho)=t g^{-1} \phi^{\prime}(\rho)$ and denote

$$
F_{\rho}=\left\{\left(r_{\rho}, \sigma\right): r_{\rho}>0, \sigma \in \Xi_{\alpha(\rho)}\right\}, \quad F_{\rho}^{R}=F_{\rho} \cap B_{R}(0),
$$

Thus $F_{\rho}^{R} \supset \Omega_{R}$ for every $\rho$ as above. 
Let $\Psi_{R}$ be the (unique) large solution of (1.1) in $B_{R}(0)$. Then $u+\Psi_{R}$ is a supersolution of (1.1) in $\Omega_{R}$ which blows up on $\partial \Omega_{R}$.

Let $w_{\alpha(\rho)}$ be the large solution of (4.27) with $\eta=\alpha(\rho), \bar{k}=\sup _{\Omega} k$ and $-\lambda=\lambda(N, p)$ as in (1.13). Then

$$
U_{\rho}\left(r_{\rho}, \sigma\right)=r_{\rho}^{-\frac{2}{\rho-1}} w_{\alpha(\rho)}(\sigma), \quad\left(r_{\rho}, \sigma\right) \in F_{\rho}
$$

is a large solution of $\Delta U-\bar{k} U^{p}=0$ in $F_{\rho}$. Since $h \geq 0$ it follows that $U_{\rho}$ is a subsolution in $\Omega_{R}$. Consequently

$$
U_{\rho} \leqq u+\Psi_{R}, \quad \text { in } \Omega_{R}, \quad \text { for } \rho \in(0, R) .
$$

Hence, setting $c(R)=\sup \Psi_{R}$ in $B_{R / 2}(0)$, we obtain

$$
u \geq \sup \left\{U_{\rho}: 0<\rho<R\right\}-c(R) \text { in } \Omega_{R / 2} .
$$

Next we observe that the condition $p \geq(N+1) /(N-3)$ implies that $\lambda \leq(N-1)(N-3) / 4$. Therefore we can apply lemma 4.7 in order to estimate $w_{\alpha(\rho)}$. Combining (4.28) with (4.29) and (4.31) we obtain (4.26).

Example. - Suppose that $\phi(\rho)=\rho^{1+\delta}$, for some $\delta>0$. Then $P_{\rho}=\left(0, \frac{\delta \rho}{1+\delta}\right)$. If we set $\rho=|z|$ then (4.26) yields, (with $\mu$ as in $4.5(a)$ ),

$$
u(z) \geq c|z|^{-2 \frac{1-\delta \mu}{p-1}}
$$

for every $z$ in $\Omega_{R}$ such that $\frac{z-P_{\rho}}{\left|z-P_{\rho}\right|} \in \Xi_{\varphi^{\prime}(\rho)}$, $0<\rho<1$. Note that, for small $\delta$, the exponent in (4.32) is close to the exponent in the upper estimate (4.24).

Inequality (4.32) implies that in the present example, if $\delta \mu<1$ then the maximal solution is in fact a large solution.

\section{APPENDIX}

This appendix is devoted to the proof of Lemmas 4.4 and 4.7. It will be assumed that $N>3$ and the notations introduced in Section 4 will be used throughout.

Let $P^{*}$ be the antipodal point to $P$ on $S^{N-1}$ and let $\mathbb{P}_{N}$ be the stereographic projection of $S^{N-1} \backslash\left\{P^{*}\right\}$ onto $\mathbb{R}^{N-1}$. It is well known that $\mathbb{P}_{N}$ is a conformal diffeomorphism with conformal factor $\varphi^{4 /(N-3)}$ where,

$$
\varphi(x)=\left(2 /\left(1+|x|^{2}\right)^{(N-3) / 2} .\right.
$$

Vol. $14, n^{\circ} 2-1997$. 
Let $w$ be a solution of (4.14) in a domain $U \subset S^{N-1} \backslash\left\{P^{*}\right\}$ and define

$$
\tilde{w}(x)=w(\sigma) \varphi(x), \quad \text { with } \sigma=\mathbb{P}_{N}^{-1}(x), \quad \forall x \in \mathbb{P}_{N}(U) .
$$

A straightforward computation (see [LP]) shows that $\tilde{u}$ satisfies the equation,

$$
\Delta \tilde{w}+\varphi^{4 /(N-3)}\left[\frac{(N-3)(N-1)}{4}-\lambda\right] \tilde{w}-\kappa \varphi^{\frac{N+1}{N-3}-p} \tilde{w}^{p}=0,
$$

in $\mathbb{P}_{N}(U)$.

Lemma A.1. - Let $w_{\eta}$ be as in Lemma 4.4. Then there exists a constant $c_{0}$, independent of $\eta$, such that (with $\tilde{w}_{\eta}$ as in (a.2)),

$$
\tilde{w}_{\eta}(y) \leq c_{0} \eta^{-2 /(p-1)}, \quad \text { for }|y|=3 \eta, \quad 0<\eta<1 .
$$

Proof. - $\tilde{w}_{\eta}$ satisfies equation (a.3) in the domain $\mathbb{P}_{N}\left(\Xi_{\eta}\right)=\{x \in$ $\left.\mathbb{R}^{N-1}:|x|>\operatorname{tg} \eta\right\}$. The function $\tilde{v}_{\eta}$ defined by,

$$
\tilde{v}_{\eta}(x)=\eta^{(N-3) / 2} \tilde{w}_{\eta}(\eta x)
$$

satisfies the equation,

$$
\Delta \tilde{v}_{\eta}+\tilde{\lambda}\left(\frac{2 n}{n^{2}+r^{2}}\right)^{2} \tilde{v}_{\eta}-\kappa\left(\frac{2 n}{n^{2}+r^{2}}\right)^{\mu / 2} \tilde{v}_{\eta}^{p}=0, \quad\left(n=\eta^{-1}\right)
$$

where $\mu=N+1-p(N-3)$ and $\tilde{\lambda}=\frac{1}{4}(N-1)(N-3)-\lambda$, in the domain $K_{\eta}=\left\{x \in \mathbb{R}^{N-1}:|x|>\eta^{-1} \operatorname{tg} \eta\right\}$. We note that for small $\eta$, $1<\eta^{-1} \operatorname{tg} \eta<2$. Therefore for $m>2$,

$$
D_{m}=\left\{x \in \mathbb{R}^{N-1}: 2<|x|<m\right\} \subset K_{\eta} \text {. }
$$

Observe that for $r=|x|>1$ and $n \geq 1$,

$$
1 / n r^{2} \leq 2 n /\left(n^{2}+r^{2}\right) \leq \min (1 / r, 2 / n)<1 .
$$

Consequently, the coefficients of $\tilde{v}_{\eta}$ and $\tilde{v}_{\eta}^{p}$ in Equation (a.6) are bounded above (in $D_{m}$ ) by $\tilde{\lambda}_{+}$and $-\beta_{n}(r)$ respectively, where

$$
\beta_{n}(r)=\kappa n^{-\mu / 2} r^{-\mu} \quad \text { if } \quad \mu \geq 0
$$

and

$$
\beta_{n}(r)=\kappa(n / 2)^{-\mu / 2} \quad \text { if } \quad \mu<0 .
$$

Let $\beta(r)=n^{\mu / 2} \beta_{n}(r)$ and consider the equation,

$$
\Delta V+\tilde{\lambda}_{+} V-\beta V^{p}=0 .
$$


If $V_{m}$ is a large solution of this equation in $D_{m}$ then $n^{-\mu / 2(1-p)} V_{m}$ is a supersolution of (a.6) in this domain and consequently,

$$
\tilde{v}_{\eta} \leq n^{-\mu / 2(1-p)} V_{m} \text { in } D_{m} \text {. }
$$

(Note that $V_{m}$ is radially symmetric.) Hence, using (a.5) and the definition of $\mu$,

$$
\tilde{w}_{\eta}(\eta x) \leq \eta^{-\frac{2}{p-1}} V_{m}(|x|), \quad \text { in } D_{m}
$$

Choosing $m>3$, (a.9) implies (a.4).

Lemma A.2. - The equation

$$
\Delta Y+\check{\lambda}\left(\frac{2}{1+r^{2}}\right)^{2} Y=0
$$

(where $\tilde{\lambda}$ is a constant and $r=|x|$ ) possesses a unique positive solution in $\mathbb{R}^{N-1} \backslash\{0\}$ such that

$$
\left.\begin{array}{c}
\lim _{r \rightarrow \infty} r^{N-3} Y=1 \\
\text { and } r^{N-3} Y \text { converges to a positive limit as } r \rightarrow 0 .
\end{array}\right\}
$$

Proof. - Consider the equation

$$
\Delta u+\left(h /\left(a+r^{2}\right)^{1+\delta}\right) u=0 \quad \text { in } \mathbb{R}^{d}, \quad d>2, \quad a>0
$$

If $\delta>0$ and $h$ is a smooth bounded function such that $h \leq(d-2)^{2} / 4$ in $\mathbb{R}^{d}$, it is known that Green's function for (a.12) is equivalent to Green's function for $\Delta$, (see [Pr, Lemma 2.4] and [Py, example 4.3.12]). Thus (a.12) possesses a positive solution in $\mathbb{R}^{d} \backslash\{0\}$ which has the same behavior at 0 and $\infty$ as Green's function of $\Delta$. This solution is unique up to a multiplicative constant. Now, if $Y$ satisfies (a.10) then the function $Z(x)=Y(x / n), n>0$, satisfies the equation

$$
\Delta Z+\tilde{\lambda}\left(\frac{2 n}{n^{2}+r^{2}}\right)^{2} Z=0 .
$$

If $n$ is chosen sufficiently small, the coefficient of $Z$ in (a.13) satisfies the conditions described above and consequently (a.10) has a solution possessing the properties stated in the lemma.

Using Lemmas A.1, A.2 we obtain,

LEMMA A.3. - Suppose that $p \geq \frac{N-1}{N-3}$. Then, for every $\tau>1$ there exists a constant $c_{1}$ depending on $N, p, \tau$ such that,

$$
w_{\eta}(\sigma) \leq c_{1}|\sigma|_{P}^{3-N} \eta^{N-3-\frac{2}{p-1}}, \quad \forall \sigma \in \Xi_{\tau \eta}, \quad 0<\eta<\pi / \tau
$$


Proof. - Let $c_{0}$ be as in Lemma A.1 and $Y$ as in Lemma A.2. In view of (a.11) we can choose $\alpha>0$ such that,

$$
\alpha Y(x) \geq c_{0}(r / 4)^{3-N} \quad \text { for } \quad r \in(0,1) .
$$

Hence by (a.4),

$$
\alpha \eta^{N-3-\frac{2}{p-1}} Y(x) \geq \tilde{w}_{\eta}(x) \quad \text { for } \quad|x|=3 \eta, \quad \eta \in(0,1) .
$$

Clearly $Y$ is a supersolution of (a.3) and $Y \rightarrow 0$ at infinity. By (a.2) $\tilde{w}_{\eta} \rightarrow 0$ at infinity. Therefore, by the maximum principle,

$$
\alpha \eta^{N-3-\frac{2}{p-1}} Y(x) \geq \tilde{w}_{\eta}(x) \quad \text { for } \quad|x| \geq 3 \eta, \quad \eta \in(0,1)
$$

This inequality together with (a.2) and (a.11) implies (a.14) for $\tau>3$. An inspection of the argument shows that the same result holds for any $\tau>1$.

Lemma A.3 provides the estimate stated in the first part of Lemma 4.4 (concerning the case $p>(N-1) /(N-3))$. When $p=(N-1) /(N-3)$ the exponent of $\eta$ in (a.14) vanishes. However in this case, a stronger estimate, namely (4.16), can be established.

Proof of Lemma 4.4 (ii). - Let

$$
Z_{\eta}(x)=w_{\eta}(\sigma), \quad \text { where } \quad x=\mathbb{P}_{N}(\sigma) .
$$

In view of (a.2), $Z_{\eta}=\varphi^{-1} \tilde{w}_{\eta}$. By $[\mathrm{GNN}] \tilde{w}_{\eta}$ is radially symmetric. Therefore, in spherical coordinates, with $r=|x|, Z_{\eta}$ satisfies the equation,

$$
Z_{\eta}^{\prime \prime}+\left[\frac{N-2}{r}+(3-N) \frac{2 r}{1+r^{2}}\right] Z_{\eta}^{\prime}-\left(\frac{2}{1+r^{2}}\right)^{2}\left(\lambda Z_{\eta}+\kappa Z_{\eta}^{p}\right)=0
$$

Claim. - For every $\bar{\eta}<\pi / 2$, there exists a constant $c_{\bar{\eta}}$ such that,

$$
Z_{\eta}(1) \leq c_{\eta}\left(\log \frac{1}{\eta}\right)^{(3-N) / 2}, \quad \text { for } \quad \eta \in(0, \bar{\eta})
$$

Note that, since $p=(N-1) /(N-3)$, point singularities for solutions of (4.14) are removable and so $\lim _{\eta \rightarrow 0} w_{\eta}=0$ in $S^{N-1} \backslash\{P\}$. Thus

$$
Z_{\eta}(1) \rightarrow 0 \quad \text { as } \eta \rightarrow 0 \text {. }
$$

Denote,

$$
H(r)=\int_{1}^{r} s^{2-N}\left(\frac{1+s^{2}}{2}\right)^{N-3} d s
$$


and let

$$
z_{\eta}(\rho)=Z_{\eta}\left(H^{-1}(\rho)\right) \quad \text { for } \quad \rho>\rho_{\eta}:=H(\operatorname{tg} \eta)
$$

Then $z_{\eta}$ satisfies the equation,

$$
z_{\eta}^{\prime \prime}=G(\rho)\left(\lambda z_{\eta}+\kappa z_{\eta}^{p}\right), \quad \rho>\rho_{\eta}
$$

where,

$$
G(H(r))=\left(\frac{2 r}{1+r^{2}}\right)^{2(N-2)}
$$

It is easily verified that,

$$
\begin{gathered}
\rho_{\eta} \eta^{N-3} \rightarrow-2^{3-N} /(N-3) \quad \text { as } \eta \rightarrow 0, \\
G(\rho)((N-3)|\rho|)^{2 \frac{N-2}{N-3}} \rightarrow 1 \quad \text { as }|\rho| \rightarrow \infty
\end{gathered}
$$

and $G(0)=1$.

Suppose that $\eta \in(0, \pi / 4)$ so that (by (a.18)) $\rho_{\eta}<0$. Let $\delta$ be a positive number (to be determined later) such that,

$$
\delta \leq \inf _{\mathbb{R}} G(\rho)(1+\rho)^{2 \frac{N-2}{N-3}}
$$

and denote by $\psi_{\eta}$ the positive solution of

$$
\left.\begin{array}{l}
\psi^{\prime \prime}=\delta(1+|\rho|)^{-2 \frac{N-2}{N-3}}\left(\lambda \psi+\kappa \psi^{p}\right) \quad \text { in }\left(-\beta_{\eta}, \beta_{\eta}\right), \\
\psi \rightarrow \infty \quad \text { as } \rho \rightarrow \pm \beta_{\eta} \quad \text { where } \quad \beta_{\eta}=-\rho_{\eta} / 2 .
\end{array}\right\}
$$

Then,

$$
z_{\eta} \leq \psi_{\eta} \quad \text { in }\left(-\beta_{\eta}, \beta_{\eta}^{-}\right)
$$

(Recall that $\lambda \geq 0$ and $\kappa>0$.) Note that (a.22) is symmetric with respect to $\rho=0$. Therefore, by uniqueness, $\psi_{\eta}$ is even and so $\psi_{\eta}^{\prime}(0)=0$. In addition we note that as $\eta \downarrow 0, \rho_{\eta} \downarrow-\infty$ so that the interval $\left(-\beta_{\eta}, \beta_{\eta}\right)$ is increasing and tends to $\mathbb{R}$. Consequently $\left\{\psi_{\eta}\right\}$ decreases with $\eta$ and because of the removable singularity result previously mentioned, $\psi_{n} \rightarrow 0$ pointwise as $\eta \rightarrow 0$. In particular,

$$
\chi_{\eta}:=\psi_{\eta}(0) \rightarrow 0 \text { as } \eta \rightarrow 0 .
$$

Denote,

$$
\varphi_{\eta}(\tau)=e^{-\tau} \psi_{\eta}(\rho) . \quad \tau=\ln (1-\rho), \quad-\beta_{\eta}<\rho \leq 0 .
$$

Then $\varphi_{\eta}$ satisfies the equation,

$$
\varphi^{\prime \prime}+\varphi^{\prime}=\delta\left(\lambda e^{(1-p) \tau} \varphi+\kappa \varphi^{p}\right) \quad \text { in }\left(0, \gamma_{\eta}\right)
$$


where $\gamma_{\eta}=\ln \left(1+\beta_{\eta}\right)$. Note that,

$$
Z_{\eta}(1)=z_{\eta}(0) \leq \chi_{\eta} \quad \text { and } \quad \varphi_{\eta}(0)=\chi_{\eta}, \quad \varphi_{\eta}^{\prime}(0)=-\chi_{\eta} . \quad \text { (a.26) }
$$

Next, we shall estimate $\chi_{\eta}$ in terms of $\beta_{\eta}$. Since $\psi_{\eta}$ is convex and its derivative vanishes at zero, $\psi_{\eta}$ attains its minimum at zero. Consequently $\varphi_{\eta}(\tau) \geq \chi_{\eta} e^{-\tau}$ and by (a.25),

$$
\varphi_{\eta}^{\prime \prime}+\varphi_{\eta}^{\prime} \geq \delta \lambda \chi_{\eta} e^{-p \tau} \quad \text { in }\left(0, \gamma_{\eta}\right)
$$

Let $y$ be the solution of

$$
y^{\prime \prime}+y^{\prime}=\delta \lambda \chi_{\eta} e^{-p \tau}, \quad y(0)=\chi_{\eta}, \quad y^{\prime}(0)=-\chi_{\eta} .
$$

Then $\left(e^{\tau} \varphi_{\eta}^{\prime}\right)^{\prime} \geq\left(e^{\tau} y^{\prime}\right)^{\prime}$ in $\left(0, \gamma_{\eta}\right)$ and $\varphi_{\eta}$ and $y$ satisfy the same initial conditions at zero. Consequently, $\varphi_{\eta} \geq y$ in $\left(0, \gamma_{\eta}\right)$. The solution of (a.27) can be explicitly computed. At this point we assume that (in addition to (a.21)) $\delta \lambda \leq p-1$. Then we obtain,

$$
\varphi_{\eta} \geq y \geq \delta \lambda \chi_{\eta} / p=: \xi_{\eta} \quad \text { in }\left(0, \gamma_{\eta}\right) .
$$

By (a.25) and (a.28),

$$
\left(e^{\tau} \varphi_{\eta}^{\prime}\right)^{\prime} \geq \delta\left(\lambda e^{(2-p) \tau} \xi_{\eta}+\kappa e^{\tau} \xi_{\eta}^{p}\right) .
$$

Hence, integrating from 0 to $\tau$ and using the fact that $\varphi_{\eta}^{\prime}(0)=-\chi_{\eta}$ we obtain,

$$
\varphi_{\eta}^{\prime}(\tau) \geq-\chi_{\eta} e^{-\tau}+\kappa \delta \xi_{\eta}^{p}\left(1-e^{-\tau}\right)
$$

Let $\tilde{c}=\frac{\kappa \delta}{2}(\delta \lambda / p)^{p}$ and $\tau_{\eta}=-\ln \left(\tilde{c} \chi_{\eta}^{p-1}\right)$. In view of (a.23) if $\eta$ is sufficiently small $\tau_{\eta}$ is positive. By (a.29), if $\bar{\tau}_{\eta}<\gamma_{\eta}$,

$$
\varphi_{\eta}^{\prime}(\tau) \geq \tilde{c} \chi_{\eta}^{p} \quad \text { for } \quad \tau \in\left[\tau_{\eta}, \gamma_{\eta}\right)
$$

Let $\varepsilon>0$ and let $\zeta$ be the solution of the equation $\zeta^{\prime}=\varepsilon \zeta^{p}$ satisfying $\zeta(0)=\xi_{\eta} / 2$. Assume that $\eta>0$ is sufficiently small so that $\zeta(0)<1$. Suppose also that $\varepsilon$ is so chosen that,

$$
\varepsilon^{2} p+\varepsilon \leq \delta \kappa \quad \text { and } \quad \varepsilon(\delta \lambda / 2 p)^{p} \leq \tilde{c}
$$

A simple computation shows that under these assumptions,

$$
\zeta^{\prime \prime}+\zeta^{\prime} \leq \delta \kappa \zeta^{p} \text { in }\left[0, \bar{\tau}_{\eta}\right]
$$

where $\bar{\tau}_{11}=\zeta^{-1}(1)=\left(\zeta(0)^{1-p}-1\right) / \varepsilon(p-1) /$ (Note that $\zeta$ is strictly increasing so that $0<\zeta<1$ in $\left(0, \bar{\tau}_{\eta}\right)$.) We also observe that for $\eta$ sufficiently small, $\tau_{\eta}<\bar{\tau}_{\eta}$. Finally, by (a.28), (a.30) and (a.31), if $\tau_{\eta}<\gamma_{\eta}$,

$$
\zeta(0) \leq \varphi_{\eta}\left(\tau_{\eta}\right) \quad \text { and } \quad \zeta^{\prime}(0) \leq \varphi_{\eta}^{\prime}\left(\tau_{\eta}\right) \text {. }
$$


Now we distinguish between two cases. Either $\gamma_{\eta} \leq \bar{\tau}_{\eta}$ for all sufficiently small $\eta$, in which case,

$$
\chi_{\eta} \leq \text { const }\left(\ln \frac{1}{\eta}\right)^{(3-N) / 2} \text { for all sufficiently small } \eta
$$

or there exists a sequence $\left\{\eta_{j}\right\}$ such that $\eta_{j} \downarrow 0$ for which $\bar{\tau}_{\eta_{j}}<\gamma_{\eta_{j}}$. In the first case, (a.26) and (a.34) imply (a.17). Therefore we consider the second case. (In what follows $\eta$ stands for an element of the sequence $\eta_{j}$.)

By (a.25) and (a.32),

$$
\left(e^{\tau} \varphi_{\eta}^{\prime}\left(\tau+\tau_{\eta}\right)\right)^{\prime} \geq\left(e^{\tau} \zeta^{\prime}(\tau)\right)^{\prime} \quad \text { for } \quad \tau \in\left(0, \bar{\tau}_{\eta}-\tau_{\eta}\right)
$$

and hence, by (a.33),

$\varphi_{n}\left(\tau+\tau_{\eta}\right) \geq \zeta(\tau), \quad \varphi_{\eta}^{\prime}\left(\tau+\tau_{\eta}\right) \geq e^{-\tau_{\eta \prime}} \zeta^{\prime}(\tau) \quad$ for $\quad \tau \in\left(0, \bar{\tau}_{\eta}-\tau_{\eta}\right)$.

Thus,

$$
\varphi_{\eta}\left(\bar{\tau}_{\eta}\right) \geq 1 \quad \text { and } \quad \varphi_{\eta}^{\prime}\left(\bar{\tau}_{\eta}\right)>0 .
$$

Finally compare the solution $\varphi_{\eta}$ of (a.25) in $\left(\bar{\tau}_{\eta}, \gamma_{\eta}\right)$ with the solution $\phi$ of

$$
\phi^{\prime \prime}+\phi^{\prime}=\delta \kappa \phi^{p} \quad \text { in }\left(\bar{\tau}_{\eta}, \gamma_{\eta}\right), \quad \phi\left(\bar{\tau}_{\eta}\right)=1, \quad \phi^{\prime}\left(\bar{\tau}_{\eta}\right)=0
$$

In view of $(\mathrm{a} .35)$,

$$
\varphi_{\eta}(\tau) \geq \phi(\tau) \text { in }\left(\bar{\tau}_{\eta}, \gamma_{\eta}\right)
$$

Now $\phi$ can be described as follows. Let $\Psi_{T}$ be the (unique) positive solution of,

$$
\left.\begin{array}{l}
\Psi^{\prime \prime}+\Psi^{\prime}=\delta \kappa \Psi^{p} \quad \text { in }(-T, T) \\
\Psi(t) \rightarrow \infty \quad \text { as }|t| \rightarrow T .
\end{array}\right\}
$$

Then $\Psi_{T}$ is even so that $\Psi_{T}^{\prime}(0)=0$ for every $T>0$. Furthermore, there exists $T_{0}$ such that $\Psi_{T_{0}}(0)=1$. (Indeed, $\Psi_{T}(t)=T^{2 /(1-p)} \Psi_{1}(t / T)$.) Consequently, $\phi(\tau)=\Psi_{T_{0}}\left(\tau-\bar{\tau}_{\eta}\right)$. Since $\phi$ blows up at $\tau=\bar{\tau}_{\eta}+T_{0}$, (a.37) implies that, $\gamma_{\eta} \leq \bar{\tau}_{\eta}+T_{0}$. Hence, (see (a.25) and (a.38)) there exist positive constants $c_{1}, c_{2}$ such that,

$$
\ln \left(1+\beta_{\eta}\right) \leq c_{1} \chi_{\eta}^{1-p}+c_{2}, \quad \text { for all sufficiently small } \eta
$$

However, by (a.20),

$$
\begin{aligned}
\ln \left(1+\beta_{\eta}\right) & =\ln \left(1-\rho_{\eta} / 2\right)=\ln \left((1+o(1)) \eta^{3-N} 2^{2-N} /(N-3)\right) \\
& =(3-N) \ln \eta+O(1)
\end{aligned}
$$


Thus,

$$
\chi_{\eta} \leq \operatorname{const}\left(\ln \frac{1}{\eta}\right)^{(3-N) / 2} \quad \text { for all sufficiently small } \eta
$$

(Recall that $1-p=2 /(3-N)$.) Now the inequality (a.17) follows from (a.26), (a.34) and (a.39). Obviously a similar inequality holds for every $r>0$ and every $0<\bar{\eta}<2 \operatorname{tg}^{-1} r$ :

$$
\left.\begin{array}{c}
Z_{\eta}(r) \leq c_{\bar{\eta}}(r)\left(\log \frac{1}{\eta}\right)^{(3-N) / 2}, \\
\text { for } \eta \in(0, \bar{\eta}) .
\end{array}\right\}
$$

Thus, for $0<\eta<\bar{\eta}<2 \operatorname{tg}^{-1} r$,

$$
\left.\begin{array}{c}
w_{\eta}(\sigma) \leq c_{\bar{\eta}}(r)\left(\log \frac{1}{\eta}\right)^{(3-N) / 2} \\
\text { for } \sigma \in\left\{\mathbb{P}_{N}^{-1}(x):|x|=r\right\} .
\end{array}\right\}
$$

By the maximum principle, the inequality holds for all $\sigma$ in $\left\{\mathbb{P}_{N}^{-1}(x)\right.$ : $|x| \geq r\}$.

Finally we turn to,

Proof of Lemma 4.7. - Define $\tilde{w}_{\eta}$ and $\tilde{v}_{\eta}$ as in Lemma A.1. Then $\tilde{v}_{\eta}$ satisfies Equation (a.6) in $K_{\eta}$. Note that,

$$
K_{\eta} \subset D_{\infty}=\left\{x \in \mathbb{R}^{N-1}: 1<|x|\right\}
$$

and that the assumptions on $p$ and $\lambda$ imply,

$$
\tilde{\lambda} \geq 0, \quad \mu<2 .
$$

For $r \geq 1$ and $n \geq 1$, the coefficient of $-\hat{v}_{\eta}$ in (a.6) is bounded above by $\gamma(r)$ where,

and

$$
\left.\begin{array}{l}
\gamma_{\eta}(r)=\bar{k}(2 \eta)^{\mu / 2} \quad \text { if } \mu \geq 0 \\
\gamma_{\eta}(r)=\bar{k} \eta^{\mu / 2} r^{-\mu} \quad \text { if } \mu<0 .
\end{array}\right\}
$$

Now consider the equation,

$$
\Delta U-\gamma_{\eta}(r) U^{p}=0
$$

Let $U_{\eta}$ be the large solution of (a.45) in $D_{\infty}$. Since $\tilde{\lambda} \geq 0, U_{\eta}$ is a subsolution of (a.6) and in view of (a.43),

$$
U_{\eta} \leq \tilde{v}_{\eta} \quad \text { in } K_{\eta} \text {. }
$$


Let $\gamma(r)=\eta^{-\mu / 2} \gamma_{\eta}(r)$ and let $U^{*}$ be the large solution of the equation,

$$
\Delta U^{*}-\gamma(r) U^{* p}=0
$$

in $D_{\infty}$. Since $\mu<2, U^{*}$ satisfies the following inequality for every $r_{0}>1$ :

$$
c^{\prime}\left(r_{0}\right)|x|^{3-N} \leq U^{*}(x) \leq c^{\prime \prime}\left(r_{0}\right)|x|^{3-N}, \quad \text { for } \quad r_{0} \leq|x|,
$$

where $c^{\prime}$ and $c^{\prime \prime}$ are positive constants (see [BM3]). Since $U^{*}$ blows up at $|x|=1$, the constant $c^{\prime}$ can be chosen to be independent of $r_{0}$. Now, $U_{\eta}=\eta^{\frac{\mu}{2(1-p)}} U^{*}$. Consequently, by (a.46) and (a.47),

$$
c^{\prime}|x|^{3-N} \eta^{\frac{\mu}{2(1-p)}} \leq \tilde{v}_{\eta}(x) \text { everywhere in } K_{\eta} \text {. }
$$

Returning to $w_{\eta}$ through (a.2) and (a.5), this inequality leads to (4.28).

\section{REFERENCES}

[BBL] R. Benguria, H. Brezis and E. Lieb, The Thomas-Fermi-Von Weizsaäcker theory of atoms and molecules, Comm. Math. Phys., Vol. 79, 1981, pp. 167-180.

[BM1] C. BANDLE and M. MARCuS, Sur les solutions maximales de problèmes elliptiques non linéaires, C. R. Acad. Sci. Paris, 311, Ser. I, 1990, pp. 91-93.

[BM2] C. BANDLE and M. MARCUS, Large solutions of semilinear elliptic equations: existence, uniqueness and asymptotic behavior, $J$ l. d'Analyse Math., Vol. 58, 1992, pp. 9-24.

[BM3] C. BANDLE and M. MARCuS, Large solutions of semilinear elliptic equations with singular coefficients, in Optimization and Nonlinear Analysis (ed. IOFFE, MARCUS, ReICH), Pitman R. N. Series, Vol. 244, 1992, pp. 25-38.

[BM4] C. BANDLE and M. MARCus, Asymptotic behavior of solutions and their derivatives for semilinear elliptic problems with blowup on the boundary, Ann. Inst. Poincaré (to appear).

[BVI BREZIS and L. VERON, Remouvable singularities for some nonlinear elliptic equations, Arch. Rat. Mech. Anal., Vol. 75, 1980, pp. 1-6.

[GNN] B. GidAs, W. Ni and L. NiREnBERG, Symmetry and related properties via the maximum principle, Comm. Math. Phys., Vol. 68, 1979, pp. 209-243.

[K] J. B. Keller, On solutions of $\Delta u=f(u)$, Comm. Pure Appl. Math., Vol. 10, 1957 , pp. 503-510.

[LP] J. N. LeE and T. H. Parker, The Yamabe problem, Bull. Amer. Math. Soc., Vol. 17, 1987, pp. 37-91.

[LG] J. F. LE GALL, A path-valued Markov process and its connections with partial differential equations, Proc. lst European Congress of Mathematics, Birkhäuser (to appear).

[M] M. MarCUS, On solutions with blow up at the boundary for a class of semilinear elliptic equations, in Developments in P.D.E. and Applications to Mathematical Physiscs, Buttazzo, Galdi and Zanghirati ed., Plenum Press, 1993, pp. 65-77.

[MV] M. MARCUS and L. VERON, Uniqueness of solutions with blow up at the boundary for a class of nonlinear elliptic equations, C. R. Acad. Sci. Paris, 317, Ser. I, 1993. pp. 559-563.

IO] R. Osserman, On the inequality $\Delta u=f(u)$, Pacific Jl. Math., Vol. 7, 1957. pp. $1641-1647$. 
[Pr] Y. PINChover, Criticality and ground states for second order elliptic equations, Jl. Diff. Equ., Vol. 80, 1989, pp. 237-250.

[Py] R. PINSKY, Positive Harmonic Functions and Diffusion, Cambridge Studies in Advanced Math., Vol. 45, 1995.

[V1] L. Veron, Semilinear elliptic equations with uniform blow up on the boundary, Jl. d'Analyse Math., Vol. 59, 1992, pp.231-250.

[V2] L. Vrron, Comportement asymptotique des solutions d'équations elliptiques semi linéaires dans $R^{N}$, Anm. Mat. Pura Appl., Vol. 127, 1981, pp. 25-50.

IV3] L. VERON, Singular solutions of some nonlinear elliptic equations, Nonlinear Anal. T. M. \& A.5, 1981, pp. 225-242.

(Manuscript received April 5, 1995.) 